\title{
Classification of Positive Ground State Solutions with Different Morse Indices for Nonlinear $N$-Coupled Schrödinger System
}

\author{
Juncheng $\mathrm{Wei}^{1, *}$ and Maoding Zhen ${ }^{2}$ \\ ${ }^{1}$ Department of Mathematics, University of British Columbia, Vancouver, BC V6T \\ 1Z2, Canada \\ 2 School of Mathematics, Hefei University of Technology, Hefei, Anhui 230009, China
}

Received 19 August 2020; Accepted (in revised version) 8 October 2020

Dedicated to Prof. Paul H. Rabinowitz with admiration on the occasion of his 80th birthday

\begin{abstract}
In this paper, we study the following $N$-coupled nonlinear Schrödinger system

$$
\left\{\begin{array}{l}
-\Delta u_{j}+u_{j}=\mu_{j} u_{j}^{3}+\sum_{i \neq j} \beta_{i, j} u_{i}^{2} u_{j}, \quad \text { in } \mathbb{R}^{n}, \\
u_{j}>0 \text { in } \mathbb{R}^{n}, u_{j}(x) \rightarrow 0 \text { as }|x| \rightarrow+\infty, j=1, \cdots, N,
\end{array}\right.
$$

where $n \leq 3, N \geq 3, \mu_{j}>0, \beta_{i, j}=\beta_{j, i}>0$ are constants and $\beta_{j, j}=\mu_{j}, j=1, \cdots, N$. There have been intensive studies for the system on existence/non-existence and classification of ground state solutions when $N=2$. However fewer results about the classification of ground state solution are available for $N \geq 3$. In this paper, we first give a complete classification result on ground state solutions with Morse indices 1 , 2 or 3 for three-coupled Schrödinger system. Then we generalize our results to $N$ coupled Schrödinger system for ground state solutions with Morse indices 1 and $N$. We show that any positive ground state solutions with Morse index 1 or Morse index $N$ must be the form of $\left(d_{1} w, d_{2} w, \cdots, d_{N} w\right)$ under suitable conditions, where $w$ is the unique positive ground state solution of certain equation. Finally, we generalize our results to fractional $N$-coupled Schrödinger system.
\end{abstract}

Key Words: Nonlinear Schrödinger system, unique ground state solution, variational method, Morse indices.

AMS Subject Classifications: 35B09, 35J47, 35J50

\section{Introduction}

In this paper, we study the following $N$-coupled nonlinear Schrödinger system

*Corresponding author. Email addresses: jcwei@math.ubc.ca (J. C. Wei), maodingzhen@163. com (M. D. Zhen) 


$$
\left\{\begin{array}{l}
-\Delta u_{j}+u_{j}=\mu_{j} u_{j}^{3}+\sum_{i \neq j} \beta_{i, j} u_{i}^{2} u_{j} \quad \text { in } \mathbb{R}^{n}, \\
u_{j}>0 \text { in } \mathbb{R}^{n}, u_{j}(x) \rightarrow 0 \text { as }|x| \rightarrow+\infty, \quad j=1, \cdots, N,
\end{array}\right.
$$

where $n \leq 3, N \geq 3, \mu_{j}>0$ are constants and $\beta_{i, j}=\beta_{j, i}>0$ are coupling parameters $\left(\beta_{j, j}=\mu_{j}\right)$. This paper is concerned with the uniqueness of ground state solution in the general case $N \geq 3$.

This system arises as standing wave solutions of the time-dependent $N$-coupled Schrödinger systems of the form

$$
\left\{\begin{array}{l}
-\sqrt{-1} \frac{\partial}{\partial t} \Phi_{j}=\Delta \Phi_{j}-V_{j}(x) \Phi_{j}+\mu_{j} \Phi_{j}\left|\Phi_{j}\right|^{2}+\sum_{i \neq j} \beta_{i, j} \Phi_{j}^{2} \Phi_{j} \quad \text { in } \mathbb{R}^{n}, \\
\Phi_{j}=\Phi_{j}(x, t) \in \mathbb{C}, \quad t>0, \quad j=1, \cdots, N,
\end{array}\right.
$$

and these systems are also known as coupled Gross-Pitaevskii equations. In the past fifteen years, a great attention has been focused on the study of two coupled systems with nonlinear terms, both for their interesting theoretical structure and their concrete applications, such as in nonlinear optics and in Bose-Einstein condensates for multi-species condensates. By using variational methods or Lyapunov-Schmidt reduction methods, there are lots of results about existence, multiplicity and qualitative properties of nontrivial solutions of two coupled elliptic system. Since it seems almost impossible for us to provided a complete list of references, we refer the readers only to [1-11,18-20,25-27] and reference therein.

For two coupled Schrödinger system with $\beta_{1,2}=\beta_{2,1}=\beta$, B. Sirakov [24] showed that if $0 \leq \beta<\min \left\{\mu_{1}, \mu_{2}\right\}$ or $\beta>\max \left\{\mu_{1}, \mu_{2}\right\}$, then $(\sqrt{k} w, \sqrt{l} w)$ is ground state solution, where $k, l$ satisfies $\mu_{1} k+\beta l=1, \mu_{1} l+\beta k=1$ and they conjecture that under the above hypotheses $(\sqrt{k} w, \sqrt{l} w)$ is the unique positive solution. For this conjecture, by the ODE method, J. Wei and W. Yao, [27, Theorem 4.2] proved this conjecture in case $\beta>\max \left\{\mu_{1}, \mu_{2}\right\}$, and [27, Theorem 4.1] proved it in case $0<\beta<\beta_{1}$, where $\beta_{1}$ is an unknown small constant. When $\beta<\min \left\{\mu_{1}, \mu_{2}\right\}, Z$. Chen and W. Zou [10] gave a complete answer to this conjecture and obtained the asymptotic behavior of ground state solution. However, the above work are for purely attractive and purely repulsive cases, there have been few results in the case of mixed couplings, i.e., the case having both positive and negative coupling constants. For the systems in the entire space with mixed couplings was considered by T. Lin and J. Wei [16], in which a 3-system was considered with two coupling constants positive and one coupling constant negative.

For $N$-coupled system with mixed couplings, J. Wei and T. Lin [16] established some general theorems for the existence and nonexistence of ground state solution and showed that when all $\beta_{i, j}$ are positive and the matrix $\mathcal{B}$ is positively definite, there exist a ground state solution which is radially symmetric. However, if all $\beta_{i, j}$ are negative, or one of $\beta_{i, j}$ is negative and the matrix $\mathcal{B}$ is positively definite, there is no ground state solution. Recently, J. Wei and Y. Wu [28] gave a systematic and an (almost) complete study on 
the existence of ground state solution for $\mathrm{N}$-coupled system when the system admits mixed couplings. By dividing this system into repulsive-mixed and total-mixed cases, they proved the nonexistence of ground state solution for repulsive-mixed case and gave an necessary condition for the existence of ground state solution for total-mixed cases. Peng et al. [21] use a construction argument for singularly perturbed elliptic problems to obtain vector solutions with some of the components synchronized between them while being segregated with the rest of the components simultaneously.

Inspired by the above-mentioned works, especially by $[14,22,28]$, in this paper our goal is two-folds. One is to give a complete classification of ground state solution with different Morse indices for three-coupled Schrödinger system under suitable conditions. Another goal of the paper is to give a different approach from [14] to get the existence of ground state solutions for N-coupled system. The difficulty is that we can't use the method introduced in [14], where the authors considered the ground state solution with Morse index $N$ on bounded domain of $\mathbb{R}^{n}$, when the parameter satisfies $-\lambda_{1}(\Omega)<\lambda_{1}=\cdots \lambda_{N}=\lambda<0$. The novelty is that in order to obtain the unique minimum point of $g\left(\tau_{1}, \cdots, \tau_{N}\right),(2.6 \mathrm{~b})$, it is not feasible to use the method in [22] by directly calculate the second derivative to determine the unique minimum values, so we use the method of Lagrange's multiplier, implicit function theorem and the Cramer's Rule to show there exists a unique $\left(\tau_{1, \min }, \cdots, \tau_{N, \min }\right), \tau_{i, \min }>0, i=1, \cdots, N$, such that $g\left(\tau_{1, \text { min }}, \cdots, \tau_{N, \text { min }}\right)=g\left(t_{1}, \cdots, t_{N}\right)_{\text {min }}$ (see details in Lemma 3.3). Then we give a complete classification of ground state solutions with Morse index 1 for system (1.1) under suitable conditions. We prove that all the ground state solutions of (1.1) must be the form of $\left(c_{1} w, \cdots, c_{N} w\right)$, where $w$ is the unique positive ground state solution of (1.5). Finally, we generalize our results to fractional $N$-coupled Schrödinger system.

Before we state our main results we introduce some notations. Let $H^{1}\left(\mathbb{R}^{n}\right)$ be the Hilbert space of functions in $\mathbb{R}^{n}$ endowed with the standard scalar product and norm

$$
\langle u, v\rangle=\int_{\mathbb{R}^{n}}(\nabla u \nabla v+u v) d x, \quad\|u\|_{H^{1}\left(\mathbb{R}^{n}\right)}^{2}=\langle u, u\rangle .
$$

The energy functional associated with (1.1) is given by

$$
E(\mathbf{u})=\sum_{j=1}^{N}\left[\frac{1}{2} \int_{\mathbb{R}^{n}}\left(\left|\nabla u_{j}\right|^{2}+u_{j}^{2}\right) d x-\frac{1}{4} \mu_{j} \int_{\mathbb{R}^{n}} u_{j}^{4} d x\right]-\frac{1}{4} \sum_{i \neq j}^{N} \beta_{i, j} u_{i}^{2} u_{j}^{2} d x .
$$

Define two Nehari manifolds

$$
\begin{gathered}
\mathbf{M}_{\mathbf{1}}=\left\{\mathbf{u} \in \mathcal{H} \backslash\{(0,0, \cdots 0,0)\}: \sum_{j=1}^{N} \int_{\mathbb{R}^{n}}\left|\nabla u_{j}\right|^{2} d x+\int_{\mathbb{R}^{n}} u_{j}^{2} d x\right. \\
\left.=\sum_{j=1}^{N} \mu_{j} \int_{\mathbb{R}^{n}} u_{j}^{4}+\sum_{i, j=1, i \neq j}^{N} \beta_{i, j} \int_{\mathbb{R}^{n}} u_{i}^{2} u_{j}^{2} d x\right\},
\end{gathered}
$$




$$
\begin{array}{r}
\mathbf{M}_{\mathbf{N}}=\left\{\mathbf{u} \in \mathcal{H} \backslash\{(0,0, \cdots 0,0)\}: \int_{\mathbb{R}^{n}}\left|\nabla u_{j}\right|^{2} d x+\int_{\mathbb{R}^{n}} u_{j}^{2} d x\right. \\
\left.=\mu_{j} \int_{\mathbb{R}^{n}} u_{j}^{4}+\sum_{i, i \neq j}^{N} \beta_{i, j} \int_{\mathbb{R}^{n}} u_{i}^{2} u_{j}^{2} d x, j=1, \cdots, N\right\},
\end{array}
$$

and the associated minimization problems

$$
c_{j}:=\inf _{\mathbf{u} \in \mathbf{M}_{\mathbf{j}}} E(\mathbf{u})=\inf _{\mathbf{u} \in \mathbf{M}_{\mathbf{j}}} \sum_{i=1}^{N} \frac{1}{4}\left[\int_{\mathbb{R}^{n}}\left|\nabla u_{i}\right|^{2} d x+\int_{\mathbb{R}^{n}} u_{i}^{2} d x\right], \quad j=1, \cdots, N,
$$

for $\mathbf{u}=\left(u_{1}, \cdots, u_{N}\right) \in \mathcal{H}$ and $\mathcal{H}=\left(H^{1}\left(\mathbb{R}^{n}\right)\right)^{N}$.

We say that $\mathbf{u}$ is a ground state solution of (1.1), if $u_{j}>0, j=1, \cdots, N$, $\mathbf{u}$ solves (1.1) and $E(\mathbf{u})=a_{i}, i=1, N$. If $\mathbf{u}$ is a nontrivial solution of (1.1), then $\mathbf{u}$ is in $\mathbf{M}_{l}, l=1, N$. It is easy to see that $\mathbf{M}_{l} \neq \varnothing$. In fact, if we take $\varphi_{j} \in \mathcal{C}_{c}^{\infty}\left(\mathbb{R}^{n}\right), j=1, \cdots, N$, with $\varphi_{j} \not \equiv 0$ and

$$
\operatorname{supp}\left(\varphi_{j}\right) \bigcap \operatorname{supp}\left(\varphi_{k}\right)=\varnothing \quad \text { for } j \neq k \text {, }
$$

then there exists $t_{1}, \cdots, t_{N}>0$, such that $\left(t_{1} \varphi_{1}, \cdots, t_{N} \varphi_{N}\right) \in \mathbf{M}_{l}$.

To state our results, we introduce the matrix $\mathcal{B}$ and $\mathcal{B}^{-}$as following, where $\mathcal{B}^{-}$is the inverse matrix of $\mathcal{B}$

$$
\mathcal{B}=\left(\begin{array}{ccc}
\beta_{1,1}, \beta_{2,1}, & \cdots & \beta_{N, 1} \\
\beta_{1,2}, \beta_{2,2}, & \cdots & \beta_{N, 2} \\
\vdots & \ddots & \vdots \\
\beta_{1, N}, \beta_{2, N}, & \cdots & \beta_{N, N}
\end{array}\right), \quad \mathcal{B}^{-}=\left(\begin{array}{ccc}
\beta^{1,1}, \beta^{1,2}, & \cdots & \beta^{1, N} \\
\beta^{2,1}, \beta^{2,2}, & \cdots & \beta^{2, N} \\
\vdots & \ddots & \vdots \\
\beta^{N, 1}, \beta^{N, 2}, & \cdots & \beta^{N, N}
\end{array}\right) .
$$

From [15], we can let $w$ be the unique positive solution of following problem

$$
\begin{cases}-\triangle u+u=u^{3} & \text { in } \mathbb{R}^{n}, \\ u>0 \text { in } \mathbb{R}^{n}, \quad u(x) \rightarrow 0 & \text { as }|x| \rightarrow+\infty .\end{cases}
$$

By Lemma 1 and Lemma 2 in [16], $w$ is also the unique positive ground solution of above problem.

\section{Statement of main results}

Before we present the results in the general case $N \geq 3$, we first explain the key ideas and main results when $N=3$.

We first study the following three-coupled nonlinear Schrödinger system

$$
\begin{cases}-\Delta u_{1}+u_{1}=\mu_{1} u_{1}^{3}+\beta_{1,2} u_{2}^{2} u_{1}+\beta_{1,3} u_{3}^{2} u_{1} & \text { in } \mathbb{R}^{n}, \\ -\Delta u_{2}+u_{2}=\mu_{2} u_{2}^{3}+\beta_{2,1} u_{1}^{2} u_{2}+\beta_{2,3} u_{3}^{2} u_{2} & \text { in } \mathbb{R}^{n}, \\ -\Delta u_{3}+u_{3}=\mu_{3} u_{3}^{3}+\beta_{3,1} u_{1}^{2} u_{3}+\beta_{3,2} u_{2}^{2} u_{3} & \text { in } \mathbb{R}^{n}, \\ u_{i}>0 \quad \text { in } \mathbb{R}^{n}, \quad u_{i}(x) \rightarrow 0 & \text { as }|x| \rightarrow+\infty, \quad i=1,2,3,\end{cases}
$$


where $n \leq 3, \mu_{j}>0$ are constants and $\beta_{i, j}=\beta_{j, i}>0, i, j=1,2,3$. By the method of moving plane, we can assume that all solutions to (2.1) are radially symmetric.

We first give an almost complete classification of ground state solution with different Morse indices for 3-coupled system under suitable conditions. We will show that any positive ground state solution with different Morse indices must be the form of $\left(c_{1} w, c_{2} w, c_{3} w\right)$, where $w$ is the unique positive ground state solution of (1.5). As far as we know, there are some results about the existence and nonexistence of ground state solution. J. Wei and Y. Wu [28] gave an (almost) complete study on the existence and nonexistence of ground state solution with different Morse indices of (2.1) under different conditions by the idea of block decomposition and measure the total interaction between different blocks for 3-coupled system when the system admits mixed couplings. For other results about the existence and nonexistence of ground state solution for threecoupled system, see $[16,17,23]$ and references therein.

We state our main results now. By the the definition of Morse index, it is well-known that if the ground state solution of (2.1) is defined on Nehari manifold $\mathbf{M}_{\mathbf{1}}$ (see(1.2a)), then the ground state solution has Morse index 1. If the ground state solution of (2.1) is defined on Nehari manifold $\mathbf{M}_{3}$ (see (1.2b)), then the ground state solution has Morse index $\leq 3$. If the ground state solution of (2.1) is defined on Nehari manifold $\mathbf{M}_{\mathbf{2}}$ defined below

$$
\begin{aligned}
\mathbf{M}_{2}=\{\mathbf{u} & \in \mathcal{H} \backslash\{(0,0,0)\}: \sum_{j=1}^{2} \int_{\mathbb{R}^{n}}\left(\left|\nabla u_{j}\right|^{2}+u_{j}^{2}\right) d x \\
= & \sum_{j=1}^{2} \mu_{j} \int_{\mathbb{R}^{n}} u_{j}^{4} d x+2 \beta_{1,2} \int_{\mathbb{R}^{n}} u_{1}^{2} u_{2}^{2} d x+\beta_{1,3} \int_{\mathbb{R}^{n}} u_{1}^{2} u_{3}^{2} d x+\beta_{2,3} \int_{\mathbb{R}^{n}} u_{2}^{2} u_{3}^{2} d x, \\
& \left.\int_{\mathbb{R}^{n}}\left(\left|\nabla u_{3}\right|^{2}+u_{3}^{2}\right) d x=\mu_{3} \int_{\mathbb{R}^{n}} u_{3}^{4} d x+\beta_{3,1} \int_{\mathbb{R}^{n}} u_{1}^{2} u_{3}^{2} d x+\beta_{3,2} \int_{\mathbb{R}^{n}} u_{2}^{2} u_{3}^{2} d x\right\},
\end{aligned}
$$

then the ground state solution has Morse index $\leq 2$. Indeed, if we consider the ground state solution on Nehari manifold $\mathbf{M}_{3}$, for any positive minimizer of $E(\mathbf{u})$ on $\mathbf{M}_{3}$, denoted by $\mathbf{v}, \mathcal{H}=\Gamma_{\mathbf{v}} \mathbf{M}_{3} \oplus\left(\mathbb{R} \mathbf{v}_{1} \times \mathbb{R} \mathbf{v}_{2} \times \mathbb{R} \mathbf{v}_{3}\right)$, where $\mathbf{v}_{1}=\left(v_{1}, 0,0\right), \mathbf{v}_{2}=\left(0, v_{2}, 0\right), \mathbf{v}_{3}=$ $\left(0,0, v_{3}\right)$ and $\Gamma_{\mathbf{v}} \mathbf{M}_{3}$ is the tangent space of $\mathbf{M}_{3}$ at $\mathbf{v}$. Since $\mathbf{v}$ is a positive minimizer of $E(\mathbf{u})$ on $\mathbf{M}_{3}$, we have $E^{\prime \prime}(\mathbf{v})(\mathbf{h}, \mathbf{h}) \geq 0$ for all $\mathbf{h} \in \Gamma_{\mathbf{v}} \mathbf{M}_{3}$, by the definition of the Morse index, we know that Morse index of $\mathbf{v}$ is less than or equals to 3 . On the other hand, since

$$
\begin{aligned}
E^{\prime \prime}(\mathbf{v})\left(\mathbf{v}_{i}, \mathbf{v}_{i}\right) & =\int_{\mathbb{R}^{n}}\left(\left|\nabla v_{i}\right|^{2}+v_{i}^{2}\right) d x-3 \mu_{i} \int_{\mathbb{R}^{n}} v_{i}^{4} d x-\sum_{j, j \neq i}^{3} \beta_{i, j} \int_{\mathbb{R}^{n}} v_{i}^{2} v_{j}^{2} d x \\
& =-2 \mu_{i} \int_{\mathbb{R}^{n}} v_{i}^{4} d x<0 \text { for all } i=1,2,3,
\end{aligned}
$$

which implies that the Morse index of $\mathbf{v}$ is greater than or equals to 3 . Thus, $\mathbf{v}$ is a ground state with Morse index 3. The other cases is similar as the proof of Morse index 3, so we omit the details here. 
For ground state solution with Morse index 1, we define a multivariate function

$$
f\left(\tau_{1}, \tau_{2}, \tau_{3}\right)=\frac{\tau_{1}^{2}+\tau_{2}^{2}+\tau_{3}^{2}}{\left(\sum_{i=1}^{3} \mu_{i} \tau_{i}^{4}+2 \sum_{i, j=1, i<j}^{3} \beta_{i, j} \tau_{i}^{2} \tau_{j}^{2}\right)^{\frac{1}{2}}} .
$$

We first prove that if $f\left(\tau_{1}, \tau_{2}, \tau_{3}\right)$ has a unique positive minimum point $\left(\tau_{1, \min }, \tau_{2, \min }, \tau_{3, \min }\right)$ under suitable conditions, then we show that $\left(\eta_{\min } \tau_{1, \min } w\right.$, $\left.\eta_{\min } \tau_{2, \min } w, \eta_{\min } \tau_{3, \min } w\right)$ is the unique positive ground state solution of (2.1) (where $\eta_{\min }$ is some positive constant defined later). For ground state solution with Morse index 3 , the key step is to show $\left(d_{1} w, d_{2} w, d_{3} w\right)$ is a ground state solution of (2.1)when matrix $\mathcal{B}$ and $\mathcal{B}^{-}$(see (1.4)) satisfy suitable conditions, where $d_{i}, i=1,2,3$ satisfy

$$
\left\{\begin{array}{l}
\beta_{11} d_{1}^{2}+\beta_{12} d_{2}^{2}+\beta_{13} d_{3}^{2}=1 \\
\beta_{21} d_{1}^{2}+\beta_{22} d_{2}^{2}+\beta_{23} d_{3}^{2}=1 \\
\beta_{31} d_{1}^{2}+\beta_{32} d_{2}^{2}+\beta_{33} d_{3}^{2}=1
\end{array}\right.
$$

Then by the same arguments as above step, we can show that if $\left(u_{1,0}, u_{2,0}, u_{3,0}\right)$ be any positive ground state solution of (2.1), then $\left(u_{1,0}, u_{2,0}, u_{3,0}\right)=\left(d_{1} w, d_{2} w, d_{3} w\right)$.

For ground state solution with Morse index 2, we first prove $\left(d_{1} w, m d_{1} w, d_{3} w\right)$ is a ground state solution of (2.1), where $d_{1}, d_{3}$ satisfy following equation,

$$
\left\{\begin{array}{l}
\left(\mu_{1}+\beta_{12} m^{2}\right) d_{1}^{2}+\beta_{13} d_{3}^{2}=1 \\
\left(\mu_{2} m^{2}+\beta_{21}\right) d_{1}^{2}+\beta_{23} d_{3}^{2}=1 \\
\left(\beta_{31}+\beta_{32} m^{2}\right) d_{1}^{2}+\mu_{3} d_{3}^{2}=1 .
\end{array}\right.
$$

Then we prove that if $\left(u_{12,0}, m u_{12,0}, u_{3,0}\right)$ be any positive ground state solution of (2.1) with Morse index 2 on Nehari manifold $\mathbf{M}_{2}$, then $\left(u_{12,0}, m u_{12,0}, u_{3,0}\right)=\left(d_{1} w, m d_{1} w, d_{3} w\right)$.

Let the matrix $\mathcal{B}$ and $\mathcal{B}^{-}$be defined at (1.4) and $f\left(\tau_{1}, \tau_{2}, \tau_{3}\right)$ be defined at (2.3). Our first result on three-component system is the following on classification of ground state solutions with Morse index 1.

Theorem 2.1. Assume $\beta_{i, j} \geq 0$, if $\left(\tau_{1,0}, \tau_{2,0}, \tau_{3,0}\right)$ satisfies $\nabla f\left(\tau_{1}, \tau_{2}, \tau_{3}\right)=\mathbf{0}$, then $\left(\eta_{0} \tau_{1,0} w\right.$, $\left.\eta_{0} \tau_{2,0} w, \eta_{0} \tau_{3,0} w\right)$ is a positive solution of $(2.1)$, where

$$
\eta_{0}=\frac{1}{\left(\beta_{i, 1} \tau_{1,0}^{2}+\beta_{i, 2} \tau_{2,0}^{2}+\beta_{i, 3} \tau_{3,0}^{2}\right)^{\frac{1}{2}}}, \quad i=1,2,3 .
$$

Conversely, under the condition that $\beta_{j}^{*}<2$, for all $j=1,2,3$ and that $\operatorname{det} \mathcal{B} \neq 0, f$ has a unique global minimum $\left(\tau_{1, \min }, \tau_{2, \min }, \tau_{3, \min }\right), \tau_{i, \min }>0, i=1,2,3$, and

$$
\left(\eta_{\min } \tau_{1, \min } w, \eta_{\min } \tau_{2, \min } w, \eta_{\min } \tau_{3, \min } w\right), \quad \tau_{i, \min }>0, \quad i=1,2,3,
$$


is the unique positive ground state solution of (2.1) with the Morse index 1, where

$$
\eta_{\min }=\frac{1}{\left(\beta_{i, 1} \tau_{1, \text { min }}^{2}+\beta_{i, 2} \tau_{2, \text { min }}^{2}+\beta_{i, 3} \tau_{3, \text { min }}^{2}\right)^{\frac{1}{2}}}, \quad i=1,2,3,
$$

and

$$
\beta_{j}^{*}=\inf \left\{\int_{\mathbb{R}^{n}}\left(|\nabla \phi|^{2}+\phi^{2}\right) d x \mid \phi \in H^{1}\left(\mathbb{R}^{n}\right), \int_{\mathbb{R}^{n}} \sum_{i=1, i \neq j}^{3} 2 \beta_{i, j} u_{i}^{2} \phi^{2} d x=1\right\} .
$$

Here, $u_{j}$ are the ground state state solution of two-coupled system. If $j=3$, then $\left(u_{1}, u_{2}\right)$ is a ground state solution of system (2.1) when $u_{3}=0$. The other cases are similar.

Furthermore all ground state solutions to (2.1) must be

$$
\left(\eta_{\min } \tau_{1, \min } w, \eta_{\min } \tau_{2, \min } w, \eta_{\min } \tau_{3, \min } w\right) .
$$

The next theorem classifies ground state solutions with Morse index 3.

Theorem 2.2. Assume $\beta_{i, j} \geq 0, \beta_{k, k}>0, \forall k, i \neq j$, det $\mathcal{B} \neq 0$ and $\sum_{i=1}^{3} \beta^{i, k} \geq 0$, for all $k=1,2,3$. Then $\left(d_{1} w, d_{2} w, d_{3} w\right)$ is a positive ground state solution of (2.1) with Morse index 3 , where $d_{i}>0$ for all $i=1,2,3$ and satisfy

$$
\left\{\begin{array}{l}
\beta_{1,1} d_{1}^{2}+\beta_{1,2} d_{2}^{2}+\beta_{1,3} d_{3}^{2}=1 \\
\beta_{2,1} d_{1}^{2}+\beta_{2,2} d_{2}^{2}+\beta_{2,3} d_{3}^{2}=1 \\
\beta_{3,1} d_{1}^{2}+\beta_{3,2} d_{2}^{2}+\beta_{3,3} d_{3}^{2}=1 .
\end{array}\right.
$$

Conversely, $\left(d_{1} w, d_{2} w, d_{3} w\right)$ is the unique positive ground state solution of (2.1) with Morse index 3.

The last result on three-component system classifies the ground state solutions with Morse index 2.

Theorem 2.3. Assume $\beta_{i, j} \geq 0, \beta_{k, k}>0, \forall k, i \neq j$, det $\mathcal{D} \neq 0, \mathbf{D}^{-}$is an inverse matrix of $\mathcal{D}$ and $\left(1+m^{2}\right) D^{11}+D^{21} \geq 0,\left(1+m^{2}\right) D^{12}+D^{22} \geq 0$. If $\left(u_{12,0}, m u_{12,0}, u_{3,0}\right)$ be any positive ground state solution of (2.1) with Morse index 2 on Nehari manifold $\mathbf{M}_{\mathbf{2}}$, then $\left(u_{12,0}, m u_{12,0}, u_{3,0}\right)=\left(d_{1} w, m d_{1} w, d_{3} w\right)$, where $d_{1}, d_{3}$ satisfy following equation,

$$
\begin{aligned}
& \left\{\begin{array}{l}
\left(\mu_{1}+\beta_{1,2} m^{2}\right) d_{1}^{2}+\beta_{1,3} d_{3}^{2}=1 \\
\left(\mu_{2} m^{2}+\beta_{2,1}\right) d_{1}^{2}+\beta_{2,3} d_{3}^{2}=1 \\
\left(\beta_{3,1}+\beta_{3,2} m^{2}\right) d_{1}^{2}+\mu_{3} d_{3}^{2}=1
\end{array}\right. \\
& \mathcal{D}=\left(\begin{array}{cc}
\mu_{1}+\mu_{2} k^{4}+2 \beta_{1,2} m^{2}, & \beta_{1,3}+\beta_{2,3} m^{2} \\
\beta_{3,1}+\beta_{3,2} m^{2}, & \mu_{3}
\end{array}\right), \quad \mathbf{D}^{-}=\left(\begin{array}{cc}
D^{11} & D^{12} \\
D^{21} & D^{22}
\end{array}\right) \text {. }
\end{aligned}
$$


Theorem 2.1 can be extended to $N$-component system as follows.

Theorem 2.4. Assume $\beta_{i, j} \geq 0$, if $\left(\tau_{1,0}, \tau_{2,0}, \cdots, \tau_{N, 0}\right)$ satisfies $\nabla g\left(\tau_{1}, \tau_{2}, \cdots, \tau_{N}\right)=\mathbf{0}$, then

$$
\left(\eta_{0} \tau_{1,0} w, \eta_{0} \tau_{2,0} w, \cdots, \eta_{0} \tau_{N, 0} w\right)
$$

is a positive solution of (1.1), where

$$
\begin{aligned}
& \eta_{0}=\frac{1}{\left(\beta_{i, 1} \tau_{1,0}^{2}+\beta_{i, 2} \tau_{2,0}^{2}+\cdots+\beta_{i, N} \tau_{N, 0}^{2}\right)^{\frac{1}{2}}}, \quad i=1, \cdots, N, \\
& g\left(\tau_{1}, \tau_{2}, \cdots, \tau_{N}\right)=\frac{\sum_{i=1}^{N} \tau_{i}^{2}}{\left(\sum_{i=1}^{N} \mu_{i} \tau_{i}^{4}+2 \sum_{i, j=1, i<j}^{N} \beta_{i, j} \tau_{i}^{2} \tau_{j}^{2}\right)^{\frac{1}{2}}} .
\end{aligned}
$$

Conversely, under the condition that $\beta_{j}^{*}<2$, for all $j=1, \cdots, N$ and that $\operatorname{det} \mathcal{B} \neq 0, g$ has a unique global minimum $\left(\tau_{1, \min }, \tau_{2, \min }, \cdots, \tau_{N, \min }\right), \tau_{i, \min }>0, i=1, \cdots, N$, and

$$
\left(\eta_{\min } \tau_{1, \min } w, \eta_{\min } \tau_{2, \min } w, \cdots \eta_{\min } \tau_{N, \min } w\right), \quad \tau_{i, \min }>0, \quad i=1, \cdots, N,
$$

is the unique positive ground state solution of (2.1) with the Morse index 1, where

$$
\begin{aligned}
& \eta_{\text {min }}=\frac{1}{\left(\beta_{i, 1} \tau_{1, \text { min }}^{2}+\beta_{i, 2} \tau_{2, \text { min }}^{2}+\cdots+\beta_{i, N} \tau_{N, \text { min }}^{2}\right)^{\frac{1}{2}}}, \quad i=1, \cdots, N, \\
& \beta_{j}^{*}=\inf \left\{\int_{\mathbb{R}^{n}}\left(|\nabla \phi|^{2}+\phi^{2}\right) d x \mid \phi \in H^{1}\left(\mathbb{R}^{n}\right), \int_{\mathbb{R}^{n}} \sum_{i=1, i \neq j}^{N} 2 \beta_{i, j} u_{i}^{2} \phi^{2} d x=1\right\} .
\end{aligned}
$$

Furthermore, all ground state solutions to (2.1) must be

$$
\left(\eta_{\min } \tau_{1, \min } w, \eta_{\min } \tau_{2, \min } w, \cdots, \eta_{\min } \tau_{N, \min } w\right) .
$$

Similarly Theorem 2.2 can be extended to $N$-component systems:

Theorem 2.5. Assume $\beta_{i, j} \geq 0, \beta_{k, k}>0, \forall k, i \neq j$, det $\mathcal{B} \neq 0$ and $\sum_{i=1}^{N} \beta^{i, k} \geq 0$, for all $k=1, \cdots, N$. Then $\left(d_{1} w, d_{2} w, \cdots, d_{N} w\right)$ is a positive ground state solution of (1.1) with Morse index $N$, where $d_{i}>0$ for all $i=1,2 \cdots, N$ and satisfy

$$
\left\{\begin{array}{l}
\beta_{1,1} d_{1}^{2}+\beta_{1,2} d_{2}^{2}+\cdots+\beta_{1, N} d_{N}^{2}=1, \\
\beta_{2,1} d_{1}^{2}+\beta_{2,2} d_{2}^{2}+\cdots+\beta_{2, N} d_{N}^{2}=1, \\
\vdots \\
\beta_{N, 1} d_{1}^{2}+\beta_{N, 2} d_{2}^{2}+\cdots+\beta_{N, N} d_{N}^{2}=1 .
\end{array}\right.
$$

Conversely $\left(d_{1} w, d_{2} w, \cdots d_{N} w\right)$ is the unique positive ground state solution of (1.1) with Morse index $N$. 
Remark 2.1. The similar results as Theorem 2.5 can be found in Guo et al. in [14], where the authors considered the ground state solution with Morse index $\mathrm{N}$ for $\mathrm{N}$-coupled system on bounded domain of $\mathbb{R}^{n}$, when the parameter satisfy $-\lambda_{1}(\Omega)<\lambda_{1}=\cdots \lambda_{N}=$ $\lambda<0$.

Remark 2.2. We should point out that Theorem 2.1 to Theorem 2.5 are also true for corresponding fractional Laplacian system, since for the following subcritical fractional equation

$$
(-\Delta)^{s} u+u=u^{q-1} \quad \text { in } \mathbb{R}^{n}
$$

R. L. Frank and E. Lenzmann [12] showed the unique positive radial least energy solutions for one dimension case and R. L. Frank, E. Lenzmann and L. Silvestre [13] showed the general unique ground state solution for dimension greater than one.

Remark 2.3. In order to obtain the unique minimum point of $g\left(\tau_{1}, \cdots, \tau_{N}\right),(2.6 \mathrm{~b})$ it is not feasible to use the method in [22] by directly calculate the second derivative to determine the unique minimum values, so we use the method of Lagrange's multiplier, implicit function theorem and the Cramer's Rule to show there exists a unique $\left(\tau_{1, \min }, \cdots, \tau_{N, \min }\right), \tau_{i, \min }>0, i=1, \cdots, N$, such that $g\left(\tau_{1, \min }, \cdots, \tau_{1, \min }\right)=$ $g\left(t_{1}, \cdots, t_{N}\right)_{\min }$ (see details in Lemma 3.3).

The paper is organized as follows. In section 3, we introduce some preliminaries that will be used to prove theorems. In Section 4, we prove Theorem 2.1 and Theorem 2.4. In Section 5, we prove Theorem 2.2 and Theorem 2.5. Finally, Theorem 2.3 will be proved in Section 6.

\section{Some preliminaries}

The energy functional associated with (2.1) is given by

$$
E(\mathbf{u})=\sum_{j=1}^{3}\left[\frac{1}{2} \int_{\mathbb{R}^{n}}\left(\left|\nabla u_{j}\right|^{2}+u_{j}^{2}\right) d x-\frac{1}{4} \mu_{j} \int_{\mathbb{R}^{n}} u_{j}^{4} d x\right]-\frac{1}{4} \sum_{i \neq j}^{3} \beta_{i, j} u_{i}^{2} u_{j}^{2} d x .
$$

Recall the Nehari manifolds $\mathbf{M}_{1}, \mathbf{M}_{2}, \mathbf{M}_{3}$ as defined in (1.2a), (1.2b) and (2.2) respectively.

Consider the minimization problems

$$
c_{i}:=\inf _{\mathbf{u} \in \mathbf{M}_{i}} E(\mathbf{u})=\inf _{\mathbf{u} \in \mathbf{M}_{i}} \sum_{j=1}^{3} \frac{1}{4}\left[\int_{\mathbb{R}^{n}}\left|\nabla u_{j}\right|^{2} d x+\int_{\mathbb{R}^{n}} u_{j}^{2} d x\right] .
$$

Define

$$
\begin{aligned}
& S=\inf _{u \in H^{1}\left(\mathbb{R}^{n}\right) \backslash\{0\}} \frac{\int_{\mathbb{R}^{n}}\left(|\nabla u|^{2}+u^{2}\right) d x}{\left(\int_{\mathbb{R}^{n}} u^{4} d x\right)^{\frac{1}{2}}}, \\
& S_{N}=\inf _{\mathbf{u} \in \mathcal{H} \backslash\{0,0, \cdots, 0\}} \frac{\sum_{i=1}^{N} \int_{\mathbb{R}^{n}}\left(\left|\nabla u_{i}\right|^{2}+u_{i}^{2}\right) d x}{\left(\sum_{i=1}^{N} \int_{\mathbb{R}^{n}} \mu_{i}\left|u_{i}\right|^{4} d x+2 \sum_{i, j=1, i<j}^{N} \beta_{i, j} \int_{\mathbb{R}^{n}} u_{i}^{2} u_{j}^{2} d x\right)^{\frac{1}{2}}} .
\end{aligned}
$$


We first have the following lemma.

Lemma 3.1. For

$$
S_{3}=f\left(\tau_{1}, \tau_{2}, \tau_{3}\right)_{\min } S, \quad S_{N}=g\left(\tau_{1}, \tau_{2}, \cdots \tau_{N}\right)_{\min } S,
$$

where

$$
f\left(\tau_{1}, \tau_{2}, \tau_{3}\right)_{\min }=\min _{\tau_{i}>0} f\left(\tau_{1}, \tau_{2}, \tau_{3}\right), \quad g\left(\tau_{1}, \cdots, \tau_{N}\right)_{\min }=\min _{\tau_{i}>0} g\left(\tau_{1}, \cdots, \tau_{N}\right) .
$$

Proof. For any $\varphi \in H^{1}\left(\mathbb{R}^{n}\right) \backslash\{0\}$, let $f\left(\tau_{1, \min }, \tau_{2, \min }, \tau_{3, \min }\right)=f\left(\tau_{1}, \tau_{2}, \tau_{3}\right)_{\min }$ and

$$
\left(u_{1}, u_{2}, u_{3}\right)=\left(\tau_{1, \min } \varphi, \tau_{2, \min } \varphi, \tau_{3, \min } \varphi\right) .
$$

Then by the definition of $S_{3}$, we have

$$
\begin{aligned}
& \frac{\sum_{i=1}^{3} \tau_{i, \text { min }}^{2}}{\left(\sum_{i=1}^{3} \mu_{i} \tau_{i, \text { min }}^{4}+2 \sum_{i, j=1, i<j}^{3} \beta_{i, j} \tau_{i, \text { min }}^{2} \tau_{j, \text { min }}^{2}\right)^{\frac{1}{2}}} \frac{\int_{\mathbb{R}^{n}}\left(|\nabla \varphi|^{2}+\varphi^{2}\right) d x}{\left(\int_{\mathbb{R}^{n}}|\varphi|^{4} d x\right)^{\frac{1}{2}}} \\
= & f\left(\tau_{1}, \tau_{2}, \tau_{3}\right)_{\min } \frac{\int_{\mathbb{R}^{n}}\left(|\nabla \varphi|^{2}+\varphi^{2}\right) d x}{\left(\int_{\mathbb{R}^{n}}|\varphi|^{4} d x\right)^{\frac{1}{2}}} \geq S_{3} .
\end{aligned}
$$

Hence

$$
f\left(\tau_{1}, \tau_{2}, \tau_{3}\right)_{\min } S \geq S_{3} .
$$

On the other hand, let $\left(u_{1, n}, u_{2, n}, u_{3, n}\right) \in \mathcal{H}$ be a minimizing sequence of $S_{3}$. Let $z_{i, n}=$ $t_{i, n} u_{i, n}, i=1,2,3$, where

$$
t_{i, n}=\left(\frac{\int_{\mathbb{R}^{n}}|w|^{4} d x}{\int_{\mathbb{R}^{n}}\left|u_{i, n}\right|^{4} d x}\right)^{\frac{1}{4}}
$$

then

$$
\int_{\mathbb{R}^{n}}\left|z_{i, n}\right|^{4} d x=\int_{\mathbb{R}^{n}}|w|^{4} d x, \quad i=1,2,3,
$$

where $w$ is the unique positive solution of Eq. (1.5). By Hölder inequality and (3.4), we have

$$
\int_{\mathbb{R}^{n}}\left|z_{i, n}\right|^{2}\left|z_{j, n}\right|^{2} d x \leq \int_{\mathbb{R}^{n}}|w|^{4} d x, \quad i, j=1,2,3 .
$$

Therefore, by (3.5) and $z_{i, n}=t_{i, n} u_{i, n}, i=1,2,3$, we can deduce that

$$
\begin{aligned}
& \frac{\sum_{i=1}^{3} \int_{\mathbb{R}^{n}}\left(\left|\nabla u_{i, n}\right|^{2}+u_{i, n}^{2}\right) d x}{\left(\sum_{i=1}^{3} \int_{\mathbb{R}^{n}}\left|u_{i, n}\right|^{4} d x+2 \sum_{i, j=1, i<j}^{3} \beta_{i, j} \int_{\mathbb{R}^{n}} u_{i, n}^{2} u_{j, n}^{2} d x\right)^{\frac{1}{2}}} \\
\geq & \frac{\sum_{i=1}^{3} \tau_{i, n}^{-2}}{\left(\sum_{i=1}^{3} \mu_{i} \tau_{i, n}^{-4}+2 \sum_{i, j=1, i<j}^{3} \beta_{i, j} \tau_{i, n}^{-2} \tau_{j, n}^{-2}\right)^{\frac{1}{2}}} \frac{\int_{\mathbb{R}^{n}}\left(|\nabla w|^{2}+w^{2}\right) d x}{\left(\int_{\mathbb{R}^{n}}|w|^{4} d x\right)^{\frac{1}{2}}} \\
\geq & f\left(\tau_{1, n}^{-1}, \tau_{2, n}^{-1}, \tau_{3, n}^{-1}\right) S \geq f\left(\tau_{1}, \tau_{2}, \tau_{3}\right)_{\min } S .
\end{aligned}
$$


Let $n \rightarrow+\infty$. We have that

$$
S_{3} \geq f\left(\tau_{1}, \tau_{2}, \tau_{3}\right)_{\min } S
$$

By (3.3) and (3.6), we get

$$
S_{3}=f\left(\tau_{1}, \tau_{2}, \tau_{3}\right)_{\min } S
$$

The case of $N \geq 4$ is similar.

To prove the existence and uniqueness of ground state solution, we study the properties of $f\left(\tau_{1}, \tau_{2}, \tau_{3}\right)$. To this end we first have following simple lemma

Lemma 3.2. If $\nabla f\left(\tau_{1}, \tau_{2}, \tau_{3}\right)=\mathbf{0}$, and

$$
\eta=\frac{1}{\left(\beta_{i, 1} \tau_{1}^{2}+\beta_{i, 2} \tau_{2}^{2}+\beta_{i, 3} \tau_{3}^{2}\right)^{\frac{1}{2}}}, \quad i=1,2,3,
$$

then $\left(\eta \tau_{1} w, \eta \tau_{2} w, \eta \tau_{3} w\right)$ is a positive solution of (2.1).

Similarly, if $\nabla g\left(\tau_{1}, \tau_{2}, \cdots, \tau_{N}\right)=\mathbf{0}$, and

$$
\eta=\frac{1}{\left(\beta_{i, 1} \tau_{1}^{2}+\beta_{i, 2} \tau_{2}^{2}+\cdots+\beta_{i, N} \tau_{N}^{2}\right)^{\frac{1}{2}}}, \quad i=1, \cdots, N,
$$

then $\left(\eta \tau_{1} w, \eta \tau_{2} w, \cdots, \eta \tau_{N} w\right)$ is a positive solution of (1.1).

Proof. By direct calculation, we have

$$
f_{\tau_{1}}\left(\tau_{1}, \tau_{2}, \tau_{3}\right)=\frac{2 \tau_{1} H_{1}\left(\tau_{1}, \tau_{2}, \tau_{3}\right)}{\left(\sum_{i=1}^{3} \mu_{i} \tau_{i}^{4}+2 \sum_{i, j=1, i<j}^{3} \beta_{i, j} \tau_{i}^{2} \tau_{j}^{2}\right)^{\frac{3}{2}}},
$$

where

$$
\begin{aligned}
H_{1}\left(\tau_{1}, \tau_{2}, \tau_{3}\right) & =\sum_{i=1}^{3} \mu_{i} \tau_{i}^{4}+2 \sum_{i, j=1, i<j}^{3} \beta_{i, j} \tau_{i}^{2} \tau_{j}^{2}-\left(\tau_{1}^{2}+\tau_{2}^{2}+\tau_{3}^{2}\right)\left(\sum_{j=1}^{3} \beta_{1, j} \tau_{j}^{2}\right) \\
& =\tau_{2}^{2}\left(\sum_{j=1}^{3}\left(\beta_{2, j}-\beta_{1, j}\right) \tau_{j}^{2}\right)+\tau_{3}^{2}\left(\sum_{j=1}^{3}\left(\beta_{3, j}-\beta_{1, j}\right) \tau_{j}^{2}\right) .
\end{aligned}
$$

Similarly, we have

$$
\left\{\begin{array}{l}
f_{\tau_{1}}\left(\tau_{1}, \tau_{2}, \tau_{3}\right)=0 \Leftrightarrow H_{1}\left(\tau_{1}, \tau_{2}, \tau_{3}\right)=0, \\
f_{\tau_{2}}\left(\tau_{1}, \tau_{2}, \tau_{3}\right)=0 \Leftrightarrow H_{2}\left(\tau_{1}, \tau_{2}, \tau_{3}\right)=0 \\
f_{\tau_{3}}\left(\tau_{1}, \tau_{2}, \tau_{3}\right)=0 \Leftrightarrow H_{3}\left(\tau_{1}, \tau_{2}, \tau_{3}\right)=0
\end{array}\right.
$$


where

$$
\begin{aligned}
& H_{2}\left(\tau_{1}, \tau_{2}, \tau_{3}\right)=\tau_{1}^{2}\left(\sum_{j=1}^{3}\left(\beta_{1, j}-\beta_{2, j}\right) \tau_{j}^{2}\right)+\tau_{3}^{2}\left(\sum_{j=1}^{3}\left(\beta_{3, j}-\beta_{2, j}\right) \tau_{j}^{2}\right) \\
& H_{3}\left(\tau_{1}, \tau_{2}, \tau_{3}\right)=\tau_{1}^{2}\left(\sum_{j=1}^{3}\left(\beta_{1, j}-\beta_{3, j}\right) \tau_{j}^{2}\right)+\tau_{2}^{2}\left(\sum_{j=1}^{3}\left(\beta_{2, j}-\beta_{3, j}\right) \tau_{j}^{2}\right) .
\end{aligned}
$$

Thus $H_{i}\left(\tau_{1}, \tau_{1}, \tau_{3}\right)=0, i=1,2,3$, which implies that

$$
\begin{cases}\sum_{j=1}^{3}\left(\beta_{2, j}-\beta_{1, j}\right) \tau_{j}^{2}=0, & \sum_{j=1}^{3}\left(\beta_{3, j}-\beta_{1, j}\right) \tau_{j}^{2}=0, \\ \sum_{j=1}^{3}\left(\beta_{1, j}-\beta_{2, j}\right) \tau_{j}^{2}=0, & \sum_{j=1}^{3}\left(\beta_{3, j}-\beta_{2, j}\right) \tau_{j}^{2}=0 \\ \sum_{j=1}^{3}\left(\beta_{1, j}-\beta_{3, j}\right) \tau_{j}^{2}=0, & \sum_{j=1}^{3}\left(\beta_{2, j}-\beta_{3, j}\right) \tau_{j}^{2}=0 .\end{cases}
$$

If $\left(\eta \tau_{1} w, \eta \tau_{2} w, \eta \tau_{3} w\right)$ is a positive solution of (2.1), then

$$
\left\{\begin{array}{l}
\beta_{1,1}\left(\eta \tau_{1}\right)^{2}+\beta_{1,2}\left(\eta \tau_{2}\right)^{2}+\beta_{1,3}\left(\eta \tau_{3}\right)^{2}=1 \\
\beta_{2,1}\left(\eta \tau_{1}\right)^{2}+\beta_{2,2}\left(\eta \tau_{2}\right)^{2}+\beta_{2,3}\left(\eta \tau_{3}\right)^{2}=1 \\
\beta_{3,1}\left(\eta \tau_{1}\right)^{2}+\beta_{3,2}\left(\eta \tau_{2}\right)^{2}+\beta_{3,3}\left(\eta \tau_{3}\right)^{2}=1
\end{array}\right.
$$

It is easy to see that (3.7) and (3.8) are equivalent. Hence, we complete the proof of Lemma 3.2. The proof in the case of $g\left(\tau_{1}, \cdots, \tau_{N}\right)$ is similar and thus omitted.

Let $\beta_{j}^{*}$ be defined at $(2.7 \mathrm{~b})$. Then we have

Lemma 3.3. Under the condition of $\beta_{j}^{*}<2$, for all $j=1,2,3$, there exists a unique $\left(\tau_{1, \min }, \tau_{2, \min }, \tau_{3, \min }\right), \tau_{i, \min }>0, i=1,2,3$ such that $f\left(\tau_{1, \min }, \tau_{2, \min }, \tau_{3, \min }\right)=f\left(t_{1}, t_{2}, t_{3}\right)_{\min }$. Similarly, under the condition of $\beta_{j}^{*}<2$, for all $j=1, \cdots, N$, there exists a unique $\left(\tau_{1, \min }, \cdots \tau_{N, \min }\right), \tau_{i, \min }>0, i=1, \cdots, N$, such that

$$
g\left(\tau_{1, \min }, \cdots, \tau_{N, \min }\right)=g\left(t_{1}, \cdots, t_{N}\right)_{\min } .
$$

Proof. Let

$$
h_{3}=\inf _{\mathcal{P}_{3}} \mathcal{G}_{3}\left(\tau_{1}, \tau_{2}, \tau_{3}\right) \text {, }
$$

where

$$
\begin{aligned}
& \mathcal{G}_{3}\left(\tau_{1}, \tau_{2}, \tau_{3}\right)=\sum_{i=1}^{3}\left(\frac{\tau_{i}^{2}}{2}-\frac{\mu_{i} \tau_{i}^{4}}{4}\right)-\frac{1}{4} \sum_{i, j=1, i<j}^{3} \beta_{i, j} \tau_{i}^{2} \tau_{j}^{2} \\
& \mathcal{P}_{3}=\left\{\left(\tau_{1}, \tau_{2}, \tau_{3}\right) \in \mathbb{R}^{3} \backslash\{(0,0,0)\} \mid \sum_{i=1}^{3}\left(\tau_{i}^{2}-\mu_{i} \tau_{i}^{4}\right)-\sum_{i, j=1, i<j}^{3} \beta_{i, j} \tau_{i}^{2} \tau_{j}^{2}=0\right\} .
\end{aligned}
$$


Then

$$
h_{3}=\inf _{\mathcal{P}_{3}} \frac{1}{4} \sum_{i=1}^{3} \tau_{i}^{2}=\inf _{\mathcal{P}_{3}} \frac{1}{4}\left(\sum_{i=1}^{3} \mu_{i} \tau_{i}^{4}+\sum_{i, j=1, i<j}^{3} \beta_{i, j} \tau_{i}^{2} \tau_{j}^{2}\right) .
$$

By a standard argument, we can see that

$$
4 h_{3}=\inf _{\left(\tau_{1}, \tau_{2}, \tau_{3}\right) \in \mathbb{R}^{3} \backslash\{(0,0,0)\}} \frac{\left(\sum_{i=1}^{3} \tau_{i}^{2}\right)^{2}}{\left(\sum_{i=1}^{3} \mu_{i} \tau_{i}^{4}+\sum_{i, j=1, i<j}^{3} \beta_{i, j} \tau_{i}^{2} \tau_{j}^{2}\right)}=f\left(\tau_{1}, \tau_{2}, \tau_{3}\right)_{\mathrm{min}}^{2} .
$$

Thus, $h_{3}$ can be attained by some $\tau_{i, \min } \geq 0$ for all $i=1,2,3$ and $\tau_{i, \min }>0$ for some $i$. By the method of Lagrange's multiplier, $\tau_{i, \min } \geq 0$ for all $i=1,2,3$ also satisfies the following system

$$
\begin{cases}\tau_{i, \min }=\mu_{i} \tau_{i, \min }^{3}+\sum_{i \neq j} \beta_{i, j} \tau_{i, \min } \tau_{j, \min }^{2} & \text { for all } i=1,2,3 \\ \tau_{i, \min } \geq 0 \quad \text { and } \quad \sum_{i=1}^{N} \tau_{i, \min }>0 & \text { for all } i=1,2,3\end{cases}
$$

Next, we prove $\tau_{i, \min }>0$ for all $i=1,2,3$.

To show that $\tau_{3, \min }>0$,we just need to prove that $c_{1}<c^{*}:=E\left(u_{1}, u_{2}, 0\right)$. In fact, by the implicit function theorem, there exists a unique

$$
t(s)=1-\frac{\int_{\mathbb{R}^{n}} \sum_{i=1}^{2} 2 \beta_{i, 3} u_{i}^{2} \phi^{2} d x-\int_{\mathbb{R}^{4}}\left(|\nabla \phi|^{2}+\phi^{2}\right) d x}{\sum_{i=1}^{2} \int_{\mathbb{R}^{4}}\left(\left|\nabla u_{i}\right|^{2}+u_{i}^{2}\right) d x} s^{2}+o\left(s^{2}\right),
$$

such that $\left(t(s) u_{1}, t(s) u_{2}, t(s) s \phi\right) \in \mathbf{M}_{1}$ for $s>0$ small enough.

Recall the definition of $\beta_{j}^{*}$ at (2.7b). $\beta_{3}^{*}$ can be attained by some $\phi_{3}^{*}$. Thus, we have

$$
t(s)=1-\frac{\left(1-\beta_{i, 3}^{*}\right) \int_{\mathbb{R}^{n}} \sum_{i=1}^{2} 2 \beta_{i, 3} u_{i}^{2}\left|\phi_{3}^{*}\right|^{2} d x}{\sum_{i=1}^{2} \int_{\mathbb{R}^{n}}\left(\left|\nabla u_{i}\right|^{2}+u_{i}^{2}\right) d x} s^{2}+o\left(s^{2}\right) \quad \text { as } s \rightarrow 0 .
$$

So,

$$
\begin{aligned}
& c_{1} \leq E\left(t u_{1}, t u_{2}, t s \phi\right) \\
= & \frac{t^{2}(s)}{4}\left(\sum_{i=1}^{2} \int_{\mathbb{R}^{n}}\left(\left|\nabla u_{i}\right|^{2}+u_{i}^{2}\right) d x+s^{2} \int_{\mathbb{R}^{n}}\left(\left|\nabla \phi_{3}^{*}\right|^{2}+\left|\phi_{3}^{*}\right|^{2}\right) d x\right) \\
= & \frac{1}{4}\left(\sum_{i=1}^{2} \int_{\mathbb{R}^{n}}\left(\left|\nabla u_{i}\right|^{2}+u_{i}^{2}\right) d x\right)-\frac{\left(2-\beta_{i, 3}^{*}\right)}{4} s^{2} \int_{\mathbb{R}^{n}} \sum_{i=1}^{2} \beta_{i, 3} u_{i}^{2}\left|\phi_{3}^{*}\right|^{2} d x \\
& \quad+o\left(s^{2}\right)<E\left(u_{1}, u_{2}, 0\right) \\
= & : c^{*}
\end{aligned}
$$


for $\beta_{3}^{*}<2$ and $s>0$ is small enough. Thus, we have $\tau_{3, \min }>0$.

Similarly we can use the condition that $\beta_{j}^{*}<2$, for all $j=1,2,3$ to show that $\tau_{i, \text { min }}>0$ for all $i=1,2,3$.

Let $s_{i}=\tau_{i, \min }^{2}$. Then (3.9) is equivalent to the following linear system

$$
\begin{cases}1=\mu_{i} s_{i}+\sum_{i \neq j} \beta_{i, j} s_{j} & \text { for all } i=1,2,3 \\ s_{i} \geq 0 & \text { for all } i=1,2,3\end{cases}
$$

By the Cramer's Rule and the fact that $\operatorname{det} \mathcal{B} \neq 0$, the linear system (3.10) has a unique solution $\mathbf{s}=\left(s_{1}, s_{2}, s_{3}\right)$.

The proof of second part of this lemma are similar to the first part of this lemma. To show $\tau_{i, \text { min }}>0$ for all $i=1, \cdots, N$, we let $m=1, \cdots, N-1$ and $\mathbf{l}_{m}=\left\{l_{1}, l_{2}, \cdots, l_{m}\right\} \subset$ $\{1, \cdots, N-1\}$ with $l_{1}<l_{2}<\cdots, l_{m}$. We define

$$
c_{\mathbf{1}_{m}, m}=\inf _{\mathbf{u} \in \mathbf{M}_{\mathbf{l}_{m}, m}} E_{\mathbf{1}_{m}, m}(\mathbf{u})=\inf _{\mathbf{u} \in \mathbf{M}_{\mathbf{l}_{m}, m}} \sum_{i=1}^{m} \frac{1}{4}\left[\int_{\mathbb{R}^{n}}\left|\nabla u_{l_{i}}\right|^{2} d x+\int_{\mathbb{R}^{n}} u_{l_{i}}^{2} d x\right],
$$

where

$$
\begin{gathered}
E_{\mathbf{1}_{m}, m}(\mathbf{u})=\sum_{i=1}^{m}\left[\frac{1}{2} \int_{\mathbb{R}^{n}}\left(\left|\nabla u_{l_{i}}\right|^{2}+u_{l_{i}}^{2}\right) d x-\frac{1}{4} \int_{\mathbb{R}^{n}} u_{l_{i}}^{4} d x\right]-\frac{1}{4} \sum_{i \neq j}^{m} \beta_{i_{j}, j} u_{l_{i}}^{2} u_{l_{j}}^{2} d x, \\
\mathbf{M}_{\mathbf{1}_{m}, m}=\left\{\mathbf{u} \in \mathcal{H} \backslash\{(0,0, \cdots 0,0)\}: \sum_{i=1}^{m} \int_{\mathbb{R}^{n}}\left|\nabla u_{l_{i}}\right|^{2} d x+\int_{\mathbb{R}^{n}} u_{l_{i}}^{2} d x\right. \\
\left.=\sum_{i=1}^{m} \mu_{i} \int_{\mathbb{R}^{n}} u_{l_{i}}^{4}+\sum_{i, j=1, i<j}^{m} 2 \beta_{i, j} \int_{\mathbb{R}^{n}} u_{l_{i}}^{2} u_{l_{j}}^{2} d x, \quad i=1, \cdots, m\right\} .
\end{gathered}
$$

If we can show

$$
\begin{aligned}
& c_{N}<c_{\mathbf{l}_{m}, m} \text { for all } m=2,3, \cdots, N-1, \\
& \mathbf{1}_{m}=\left\{l_{1}, l_{2}, \cdots, l_{m}\right\} \subset\{1, \cdots, N-1\},
\end{aligned}
$$

with

$$
l_{1}<l_{2}<\cdots, l_{m}
$$

then we can see that $\tau_{i, \min }>0$ for all $i=1, \cdots, N$. Without loss of generality, we assume $c_{N-1}=\min \left\{c_{\mathbf{1}_{m}, m}\right\}$ and it is attained by $\left(u_{1}, u_{2}, \cdots, u_{N-1}\right)$. By the implicit function theorem, there exists a unique

$$
t(s)=1-\frac{\int_{\mathbb{R}^{4}} \sum_{i=1}^{N-1} 2 \beta_{i, N} u_{i}^{2} \phi^{2} d x-\int_{\mathbb{R}^{4}}\left(|\nabla \phi|^{2}+\phi^{2}\right) d x}{\sum_{i=1}^{N-1} \int_{\mathbb{R}^{4}}\left(\left|\nabla u_{i}\right|^{2}+u_{i}^{2}\right) d x} s^{2}+o\left(s^{2}\right),
$$


such that $\left(t(s) u_{1}, t(s) u_{2}, \cdots, t(s) u_{N-1}, t(s) s \phi\right) \in \mathbf{M}_{1}$ for $s>0$ small enough. So,

$$
\begin{aligned}
& E_{N}\left(t u_{1}, t u_{2}, \cdots, t u_{N-1}, t s \phi\right) \\
= & \frac{t^{2}(s)}{4}\left(\sum_{i=1}^{N-1} \int_{\mathbb{R}^{4}}\left(\left|\nabla u_{i}\right|^{2}+u_{i}^{2}\right) d x+s^{2} \int_{\mathbb{R}^{4}}\left(\left|\nabla \phi_{N}^{*}\right|^{2}+\left|\phi_{N}^{*}\right|^{2}\right) d x\right) \\
= & \frac{1}{4}\left(\sum_{i=1}^{N-1} \int_{\mathbb{R}^{4}}\left(\left|\nabla u_{i}\right|^{2}+u_{i}^{2}\right) d x\right)-\frac{\left(2-\beta_{i, N}^{*}\right)}{4} s^{2} \int_{\mathbb{R}^{4}} \sum_{i=1}^{N-1} 2 \beta_{i, N} u_{i}^{2}\left|\phi_{N}^{*}\right|^{2} d x \\
& \quad+o\left(s^{2}\right) \\
< & E_{N-1}\left(u_{1}, u_{2}, \cdots, u_{N-1}\right),
\end{aligned}
$$

since $\beta_{i, N}^{*}<2$ and $s>0$ is small enough. Thus, we have $\tau_{N, \min }>0$. The other cases are similar.

\section{Proof of Theorem 2.1 and Theorem 2.4}

Proof of Theorem 2.1 and Theorem 2.4. By Lemma 3.2, $\left(\eta_{0} \tau_{1,0} w, \eta_{0} \tau_{2,0} w, \eta_{0} \tau_{3,0} w\right)$ is a positive solution of (2.1), where

$$
\eta_{0}=\frac{1}{\left(\beta_{i 1} \tau_{1,0}^{2}+\beta_{i 2} \tau_{2,0}^{2}+\beta_{i, 3} \tau_{3,0}^{2}\right)^{\frac{1}{2}}}, \quad i=1,2,3,
$$

and $\left(\tau_{1,0}, \tau_{2,0}, \tau_{3,0}\right)$ satisfies $\nabla f\left(\tau_{1}, \tau_{2}, \tau_{3}\right)=\mathbf{0}$.

Next, we show that $\left(\eta_{\min } \tau_{1, \min } w, \eta_{\min } \tau_{2, \operatorname{mim}} w, \eta_{\min } \tau_{3, \min } w\right)$ is the unique positive ground state solution of (2.1), where

$$
\eta_{\min }=\frac{1}{\left(\beta_{i 1} \tau_{1, \text { min }}^{2}+\beta_{i 2} \tau_{2, \text { min }}^{2}+\beta_{i, 3} \tau_{3, \text { min }}^{2}\right)^{\frac{1}{2}}}, \quad i=1,2,3 \text {, }
$$

and $\left(\tau_{1, \min }, \tau_{2, \min }, \tau_{3, \min }\right)$ satisfies

$$
f\left(\tau_{1, \min }, \tau_{2, \min }, \tau_{3, \min }\right)=f\left(\tau_{1}, \tau_{2}, \tau_{3}\right)_{\min }
$$

Since $\left(\eta_{\min } \tau_{1, \min } w, \eta_{\min } \tau_{2, \min } w, \eta_{\min } \tau_{3, \min } w\right)$ is a positive solution of (2.1), we have

$$
\begin{aligned}
& \left(\eta_{\min } \tau_{1, \min } w, \eta_{\min } \tau_{2, \min } w, \eta_{\min } \tau_{3, \min } w\right) \in \mathbf{M}_{1}, \\
& \frac{1}{4} \int_{\mathbb{R}^{n}}\left(|\nabla w|^{2}+w^{2}\right) d x=\frac{1}{4} S^{2} .
\end{aligned}
$$


Since $\nabla f\left(\tau_{1, \min }, \tau_{2, \min }, \tau_{3, \min }\right)=\mathbf{0}$, from (3.7) in Lemma 3.2, we have

$$
\begin{cases}\sum_{j=1}^{3}\left(\beta_{2, j}-\beta_{1, j}\right) \tau_{j, \text { min }}^{2}=0, & \sum_{j=1}^{3}\left(\beta_{3, j}-\beta_{1, j}\right) \tau_{j, \text { min }}^{2}=0 \\ \sum_{j=1}^{3}\left(\beta_{1, j}-\beta_{2, j}\right) \tau_{j, \text { min }}^{2}=0, & \sum_{j=1}^{3}\left(\beta_{3, j}-\beta_{2, j}\right) \tau_{j, \text { min }}^{2}=0 \\ \sum_{j=1}^{3}\left(\beta_{1, j}-\beta_{3, j}\right) \tau_{j, \text { min }}^{2}=0, & \sum_{j=1}^{3}\left(\beta_{2, j}-\beta_{3, j}\right) \tau_{j, \text { min }}^{2}=0 .\end{cases}
$$

Thus, by (4.1) and direct calculation, we have

$$
\begin{aligned}
& \sum_{i=1}^{3} \mu_{i} \tau_{i, \min }^{4}+2 \sum_{i, j=1, i<j}^{3} \beta_{i, j} \tau_{i, \min }^{2} \tau_{j, \min }^{2} \\
= & \tau_{1, \min }^{2}\left(\beta_{1,1} \tau_{1, \min }^{2}+\beta_{1,2} \tau_{2, \min }^{2}+\beta_{1,3} \tau_{3, \min }^{2}\right) \\
& \quad+\tau_{2, \min }^{2}\left(\beta_{2,1} \tau_{1, \min }^{2}+\beta_{2,2} \tau_{2, \min }^{2}+\beta_{2,3} \tau_{3, \min }^{2}\right) \\
& \quad+\tau_{3, \min }^{2}\left(\beta_{3,1} \tau_{1, \min }^{2}+\beta_{3,2} \tau_{2, \min }^{2}+\beta_{3,3} \tau_{3, \min }^{2}\right) \\
= & \sum_{i=1}^{3} \tau_{i, \min }^{2}\left(\beta_{3,1} \tau_{1, \min }^{2}+\beta_{3,2} \tau_{2, \min }^{2}+\beta_{3,3} \tau_{3, \min }^{2}\right) \\
= & \sum_{i=1}^{3} \tau_{i, \min }^{2}\left(\beta_{2,1} \tau_{1, \min }^{2}+\beta_{2,2} \tau_{2, \min }^{2}+\beta_{2,3} \tau_{3, \min }^{2}\right) \\
= & \sum_{i=1}^{3} \tau_{i, \min }^{2}\left(\beta_{1,1} \tau_{1, \min }^{2}+\beta_{1,2} \tau_{2, \min }^{2}+\beta_{1,3} \tau_{3, \min }^{2}\right) .
\end{aligned}
$$

So

$$
\begin{aligned}
& =\frac{f\left(\tau_{1, \min }, \tau_{2, \min }, \tau_{3, \min }\right)}{\left(\sum_{i=1}^{3} \mu_{i} \tau_{i, \min }^{4}+2 \sum_{i, j=1, i<j}^{3} \beta_{i, j} \tau_{i, \min }^{2} \tau_{j, \text { min }}^{2}\right)^{\frac{1}{2}}} \\
& =\frac{\left(\sum_{i=1}^{3} \tau_{i, \min }^{2}\right)^{\frac{1}{2}}}{\left(\beta_{3,1} \tau_{1, \text { min }}^{2}+\beta_{3,2} \tau_{2, \text { min }}^{2}+\beta_{3,3} \tau_{3, \text { min }}^{2}\right)^{\frac{1}{2}}} .
\end{aligned}
$$

On the one hand, for any $\left(u_{1}, u_{2}, u_{3}\right) \in \mathbf{M}_{1}$ and by the definition of $S_{3}$ (see (3.2b)), we have

$$
\frac{1}{4} \sum_{i=1}^{3} \int_{\mathbb{R}^{n}}\left(\left|\nabla u_{i}\right|^{2}+u_{i}^{2}\right) d x \geq \frac{1}{4} S_{3}^{2} .
$$


Hence

$$
c \geq \frac{1}{4} S_{3}^{2}
$$

On the other hand, by (4.2) and $\left(\eta_{\min } \tau_{1, \min } w, \eta_{\min } \tau_{2, \min } w, \eta_{\min } \tau_{3, \min } w\right) \in \mathbf{M}_{1}$, we have

$$
\begin{aligned}
c & \leq E\left(\eta_{\min } \tau_{1, \min } w, \eta_{\min } \tau_{2, \min } w, \eta_{\min } \tau_{3, \min } w\right) \\
& =\frac{1}{4}\left(\sum_{i=1}^{3} \tau_{i, \min }^{2}\right) \eta^{2} \int_{\mathbb{R}^{n}}\left(|\nabla w|^{2}+w^{2}\right) d x \\
& =\frac{1}{4} \frac{\sum_{i=1}^{3} \tau_{i, \min }^{2}}{\beta_{i, 1} \tau_{1, \min }^{2}+\beta_{i, 2} \tau_{2, \min }^{2}+\beta_{i, 3} \tau_{3, \min }^{2}} \int_{\mathbb{R}^{n}}\left(|\nabla w|^{2}+w^{2}\right) d x \\
& =\frac{\sum_{i=1}^{3} \tau_{i, \min }^{2}}{\beta_{i, 1} \tau_{1, \min }^{2}+\beta_{i 2} \tau_{2, \min }^{2}+\beta_{i, 3} \tau_{3, \min }^{2}} \frac{1}{4} S^{2} \\
& =\frac{1}{4} f\left(\tau_{1, \min }, \tau_{2, \min }, \tau_{3, \min }\right)^{2} S^{2}=\frac{1}{4} S_{3}^{2} .
\end{aligned}
$$

So,

$$
E\left(\eta_{\min } \tau_{1, \min } w, \eta_{\min } \tau_{2, \min } w, \eta_{\min } \tau_{3, \min } w\right)=\frac{1}{4} S_{3}^{2}
$$

Consequently, $\left(\eta_{\min } \tau_{1, \min } w, \eta_{\min } \tau_{2, \min } w, \eta_{\min } \tau_{3, \min } w\right)$ is a positive ground state solution of (2.1). If $\left(\tau_{1, \min }, \tau_{2, \min }, \tau_{3, \min }\right)$ is the unique minimum point of $f\left(\tau_{1}, \tau_{2}, \tau_{3}\right)$, then $\left(\eta_{\min } \tau_{1, \min } w, \eta_{\min } \tau_{2, \min } w, \eta_{\min } \tau_{3, \min } w\right)$ is the unique positive ground state solution of (2.1) of the form $\left(c_{1} w, c_{2} w, c_{3} w\right)$.

By Lemma 3.3 and above arguments, we complete the proof. The proof of Theorem 2.4 are similar to Theorem 2.1, we only need to replace $i=3$ to $i=N$ and use the second part of Lemma 3.2, we omit the details here. Next, we prove the second part of Theorem 2.1 and Theorem 2.4 .

Let $\left(\eta_{\min } \tau_{1, \min } w, \eta_{\min } \tau_{2, \min } w, \eta_{\min } \tau_{3, \min } w\right)$ be the unique positive ground state solution of $(2.1)$ of the form $\left(c_{1} w, c_{2} w, c_{3} w\right)$ and let $\left(u_{1,0}, u_{2,0}, u_{3,0}\right)$ be any positive ground state solution of (2.1) of Morse index 1. We first claim that

$$
\begin{array}{ll}
\int_{\mathbb{R}^{n}}\left|u_{i, 0}\right|^{4} d x=\eta_{\min }^{4} \tau_{i, \min }^{4} \int_{\mathbb{R}^{n}}|w|^{4} d x, & i=1,2,3, \\
\int_{\mathbb{R}^{n}}\left|u_{i, 0}\right|^{2}\left|u_{j, 0}\right|^{2} d x=\eta_{\min }^{4} \tau_{i, \min }^{2} \tau_{j, \min }^{2} \int_{\mathbb{R}^{n}}|w|^{4} d x & \text { for } i, j=1,2,3 .
\end{array}
$$

To prove (4.3a) and (4.3b), we use implicit function theorem. We first consider the following 3-coupled system, where $\mu_{1}$ is replaced by $\mu$ in system (2.1):

$$
\begin{cases}-\Delta u_{1}+u_{1}=\mu u_{1}^{3}+\beta_{1,2} u_{2}^{2} u_{1}+\beta_{1,3} u_{3}^{2} u_{1} & \text { in } \mathbb{R}^{n}, \\ -\Delta u_{2}+u_{2}=\mu_{2} u_{2}^{3}+\beta_{2,1} u_{1}^{2} u_{2}+\beta_{2,3} u_{3}^{2} u_{2} & \text { in } \mathbb{R}^{n}, \\ -\Delta u_{3}+u_{3}=\mu_{3} u_{3}^{3}+\beta_{3,1} u_{1}^{2} u_{3}+\beta_{3,2} u_{2}^{2} u_{3} & \text { in } \mathbb{R}^{n}, \\ u_{i}>0 \quad \text { in } \mathbb{R}^{n}, \quad u_{i}(x) \rightarrow 0 & \text { as }|x| \rightarrow+\infty, \quad i=1,2,3,\end{cases}
$$


and the following function

$$
f^{\mu}\left(\mu, \tau_{1}, \tau_{2}, \tau_{3}\right)=\frac{\tau_{1}^{2}+\tau_{2}^{2}+\tau_{3}^{2}}{\left(\mu \tau_{1}^{4}+\sum_{i=2}^{3} \mu_{i} \tau_{i}^{4}+2 \sum_{i, j=1, i<j}^{3} \beta_{i, j} \tau_{i}^{2} \tau_{j}^{2}\right)^{\frac{1}{2}}} .
$$

By the similar arguments as Lemma 3.2 and Lemma 3.3, there exists a small $0<\epsilon<1$ such that $\left(\tau_{1, \min }^{*}(\mu), \tau_{2, \min }^{*}(\mu), \tau_{3, \min }^{*}(\mu)\right)$ is unique for $\mu \in\left(\mu_{1}-\epsilon, \mu_{1}+\epsilon\right)$ and

$$
\left(\tau_{1, \min }^{*}(\mu), \tau_{2, \min }^{*}(\mu), \tau_{3, \min }^{*}(\mu)\right)
$$

satisfies

$$
f_{\tau_{i}}^{\mu}\left(\mu, \tau_{1}, \tau_{2}, \tau_{3}\right)=\frac{2 \tau_{i} H_{i}^{\mu}\left(\mu, \tau_{1}, \tau_{2}, \tau_{3}\right)}{\left(\mu \tau_{1}^{4}+\sum_{i=2}^{3} \mu_{i} \tau_{i}^{4}+2 \sum_{i, j=1, i<j}^{3} \beta_{i, j} \tau_{i}^{2} \tau_{j}^{2}\right)^{\frac{3}{2}}}=0, \quad i=1,2,3,
$$

where

$$
\begin{aligned}
& H_{1}^{\mu}\left(\mu, \tau_{1}, \tau_{2}, \tau_{3}\right)=\sum_{i=1}^{3} \mu_{i} \tau_{i}^{4}+2 \sum_{i, j=1, i<j}^{3} \beta_{i, j} \tau_{i}^{2} \tau_{j}^{2}-\left(\tau_{1}^{2}+\tau_{2}^{2}+\tau_{3}^{2}\right)\left(\sum_{j=1}^{3} \beta_{1, j} \tau_{j}^{2}\right) \\
& =\tau_{2}^{2}\left(\left(\beta_{2,1}-\mu\right) \tau_{1}^{2}+\sum_{j=2}^{3}\left(\beta_{2, j}-\beta_{1, j}\right) \tau_{j}^{2}\right)+\tau_{3}^{2}\left(\left(\beta_{3,1}-\mu\right) \tau_{1}^{2}+\sum_{j=2}^{3}\left(\beta_{3, j}-\beta_{1, j}\right) \tau_{j}^{2}\right), \\
& H_{2}^{\mu}\left(\mu, \tau_{1}, \tau_{2}, \tau_{3}\right)=\tau_{1}^{2}\left(\left(\mu-\beta_{2,1}\right) \tau_{1}^{2}+\sum_{j=2}^{3}\left(\beta_{1, j}-\beta_{2, j}\right) \tau_{j}^{2}\right)+\tau_{3}^{2}\left(\sum_{j=1}^{3}\left(\beta_{3, j}-\beta_{2, j}\right) \tau_{j}^{2}\right), \\
& H_{3}^{\mu}\left(\mu, \tau_{1}, \tau_{2}, \tau_{3}\right)=\tau_{1}^{2}\left(\left(\mu-\beta_{3,1}\right) \tau_{1}^{2}+\sum_{j=2}^{3}\left(\beta_{1, j}-\beta_{3, j}\right) \tau_{j}^{2}\right)+\tau_{2}^{2}\left(\sum_{j=1}^{3}\left(\beta_{2, j}-\beta_{3, j}\right) \tau_{j}^{2}\right) .
\end{aligned}
$$

Thus $H_{i}^{\mu}\left(\mu, \tau_{1}, \tau_{2}, \tau_{3}\right)=0, i=1,2,3$, which implies that

$$
\left\{\begin{array}{l}
\left(\beta_{2,1}-\mu\right) \tau_{1}^{2}+\sum_{j=2}^{3}\left(\beta_{2, j}-\beta_{1, j}\right) \tau_{j}^{2}=0, \\
\left(\beta_{3,1}-\mu\right) \tau_{1}^{2}+\sum_{j=2}^{3}\left(\beta_{3, j}-\beta_{1, j}\right) \tau_{j}^{2}=0, \\
\left(\mu-\beta_{2,1}\right) \tau_{1}^{2}+\sum_{j=2}^{3}\left(\beta_{1, j}-\beta_{2, j}\right) \tau_{j}^{2}=0, \\
\left(\mu-\beta_{3,1}\right) \tau_{1}^{2}+\sum_{j=2}^{3}\left(\beta_{1, j}-\beta_{3, j}\right) \tau_{j}^{2}=0, \\
\sum_{j=1}^{3}\left(\beta_{3, j}-\beta_{2, j}\right) \tau_{j}^{2}=0, \quad \sum_{j=1}^{3}\left(\beta_{2, j}-\beta_{3, j}\right) \tau_{j}^{2}=0 .
\end{array}\right.
$$


Since $\left(\tau_{1, \min }, \tau_{2, \min }, \tau_{3, \min }\right)$ is the minimum point of $f\left(\tau_{1}, \tau_{2}, \tau_{3}\right)$ (see (2.3)), we have

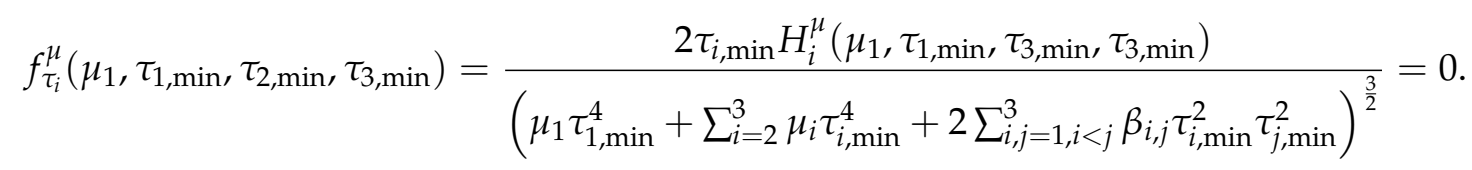

By direct calculation, we have

$$
f_{\tau_{i} \tau_{j}}^{\mu}\left(\mu_{1}, \tau_{1, \min }, \tau_{2, \min }, \tau_{3, \min }\right)=\frac{G_{i, j}}{\left(\mu_{1} \tau_{1, \min }^{4}+\sum_{i=2}^{3} \mu_{i} \tau_{i, \min }^{4}+2 \sum_{i, j=1, i<j}^{3} \beta_{i, j} \tau_{i, \min }^{2} \tau_{j, \min }^{2}\right)^{\frac{3}{2}}},
$$

where

$$
G_{i, j}=4 \tau_{i, \min } \tau_{j, \min }\left(\sum_{k=1}^{3}\left(2 \beta_{j, k}-\beta_{i, k}-\beta_{i, j}\right) \tau_{k, \min }^{2}\right) .
$$

By (4.6), we have

$$
G_{i, j}=4 \tau_{i, \min } \tau_{j, \min }\left(\sum_{k=1}^{3}\left(\beta_{j, k}-\beta_{i, j}\right) \tau_{k, \min }^{2}\right) .
$$

Since $\left(\tau_{i, \min }, \tau_{2, \min }, \tau_{3, \min }\right)$ is the minimum point of $f\left(\tau_{1}, \tau_{2}, \tau_{3}\right)$ (see $\left.(2.3)\right)$, then

$$
f_{\tau_{i}}^{\mu}\left(\mu_{1}, \tau_{1, \min }, \tau_{2, \min }, \tau_{3, \min }\right)=0, \quad i=1,2,3
$$

Let $\mathcal{G}=\left[G_{i, j}\right]_{3 \times 3}$ be the matrix defined above, then $\operatorname{det} \mathcal{G} \neq 0$ and so

$$
\nabla f_{\tau_{i}}^{\mu}\left(\mu_{1}, \tau_{1, \min }, \tau_{2, \min }, \tau_{3, \min }\right) \neq 0, \quad i, j=1,2,3 .
$$

By the implicit function theorem, we know

$$
\left(\tau_{1, \min }^{*}(\mu), \tau_{2, \min }^{*}(\mu), \tau_{3, \min }^{*}(\mu)\right)
$$

is also $C^{1}$ for $\mu \in\left(\mu_{1}-\epsilon, \mu_{1}+\epsilon\right)$. Thus, the energy functional associated with (4.4) is given by

$$
\begin{aligned}
E(\mu, \mathbf{u})= & \sum_{j=1}^{3} \frac{1}{2} \int_{\mathbb{R}^{n}}\left(\left|\nabla u_{j}\right|^{2}+u_{j}^{2}\right) d x-\sum_{j=2}^{3} \frac{1}{4} \mu_{j} \int_{\mathbb{R}^{n}} u_{j}^{4} d x-\frac{1}{4} \mu \int_{\mathbb{R}^{n}} u_{1}^{4} d x \\
& \quad-\frac{1}{4} \int_{\mathbb{R}^{n}} \sum_{i \neq j}^{3} \beta_{i, j} u_{i}^{2} u_{j}^{2} d x, \\
c_{1}(\mu):= & E\left(\mu, \eta_{\min }^{*} \tau_{1, \min }^{*}(\mu) w, \eta_{\min }^{*} \tau_{2, \min }^{*}(\mu) w, \eta_{\min }^{*} \tau_{3, \min }^{*}(\mu) w\right) \\
= & \sum_{j=1}^{3}\left(\eta_{\min }^{*}\right)^{2}\left(\tau_{j, \min }^{*}\right)^{2} \frac{1}{4}\left[\int_{\mathbb{R}^{n}}|\nabla w|^{2} d x+\int_{\mathbb{R}^{n}} w^{2} d x\right] .
\end{aligned}
$$


Next, we show that

$$
c_{1}(\mu)=\inf _{\left(u_{1}, u_{2}, u_{3}\right) \in \mathcal{H} \backslash\{(0,0,0)\}} \max _{t>0} E\left(\mu, t u_{1}, t u_{2}, t u_{3}\right) .
$$

Indeed, by the definition of $c_{1}(\mu)$, for any $\epsilon>0$, we can take a $\left(u_{1}, u_{2}, u_{3}\right) \not \equiv(0,0,0)$, such that

$$
\sum_{j=1}^{3} \int_{\mathbb{R}^{n}}\left(\left|\nabla u_{j}\right|^{2}+u_{j}^{2}\right) d x=\sum_{j=2}^{3} \mu_{j} \int_{\mathbb{R}^{n}} u_{j}^{4} d x+\frac{1}{4} \mu \int_{\mathbb{R}^{n}} u_{1}^{4} d x+\int_{\mathbb{R}^{n}} \sum_{i \neq j}^{3} \beta_{i, j} u_{i}^{2} u_{j}^{2} d x
$$

and

$$
E(\mu, \mathbf{u})<c_{1}(\mu)+\epsilon
$$

By (4.8), we have

$$
E(\mu, \mathbf{u})=\max _{t>0} E\left(\mu, t u_{1}, t u_{2}, t u_{3}\right) \geq \inf _{\left(u_{1}, u_{2}, u_{3}\right) \in \mathcal{H} \backslash\{(0,0,0)\}} \max _{t>0} E\left(\mu, t u_{1}, t u_{2}, t u_{3}\right) .
$$

Thus,

$$
c_{1}(\mu) \geq \inf _{\left(u_{1}, u_{2}, u_{3}\right) \in \mathcal{H} \backslash\{(0,0,0)\}} \max _{t>0} E_{\mu}\left(t u_{1}, t u_{2}, t u_{3}\right) .
$$

On the other hand, by the definition of

$$
\inf _{\left(u_{1}, u_{2}, u_{3}\right) \in \mathcal{H} \backslash\{(0,0,0)\}} \max _{t>0} E\left(\mu, t u_{1}, t u_{2}, t u_{3}\right) \quad \text { for any } \epsilon>0,
$$

we can take a $\left(u_{1}, u_{2}, u_{3}\right) \not \equiv(0,0,0)$ such that

$$
\begin{aligned}
& E\left(\mu, t_{\mathbf{u}} u_{1}, t_{\mathbf{u}} u_{2}, t_{\mathbf{u}} u_{3}\right)=\max _{t>0} E\left(\mu, t u_{1}, t u_{2}, t u_{3}\right) \\
< & \inf _{\left(u_{1}, u_{2}, u_{3}\right) \in \mathcal{H} \backslash\{(0,0,0)\}} \max _{t>0} E\left(\mu, t u_{1}, t u_{2}, t u_{3}\right)+\epsilon
\end{aligned}
$$

and $\left(t_{\mathbf{u}} u_{1}, t_{\mathbf{u}} u_{2}, t_{\mathbf{u}} u_{3}\right)$ satisfies (4.8), which implies that

$$
\left(t_{\mathbf{u}} u_{1}, t_{\mathbf{u}} u_{2}, t_{\mathbf{u}} u_{3}\right) \in \mathbf{M}_{1, \mu}
$$

where $\mathbf{M}_{1, \mu}$ is a Nehari manifold when $\mu_{1}$ was replaced by $\mu$ in Nehari manifold $\mathbf{M}_{1}$. Thus

$$
c_{1}(\mu) \leq \inf _{\left(u_{1}, u_{2}, u_{3}\right) \in \mathcal{H} \backslash\{(0,0,0)\}} \max _{t>0} E_{\mu}\left(t u_{1}, t u_{2}, t u_{3}\right) .
$$

So the proof of (4.7) is complete. Thus, there exists $t(\mu)>0$ such that

$$
E\left(\mu, t(\mu) u_{1,0}, t(\mu) u_{2,0}, t(\mu) u_{3,0}\right)=\max _{t>0} E\left(\mu, t u_{1,0}, t u_{2,0}, t u_{3,0}\right),
$$


where $t(\mu)>0$ satisfies $F(\mu, t(\mu))=0$ and

$$
\begin{aligned}
F(\mu, t)=t^{2} & \left(\mu \int_{\mathbb{R}^{n}} u_{1,0}^{4} d x+\sum_{j=2}^{3} \mu_{j} \int_{\mathbb{R}^{n}} u_{j, 0}^{4} d x+\int_{\mathbb{R}^{n}} \sum_{i \neq j}^{3} \beta_{i, j} u_{i, 0}^{2} u_{j, 0}^{2} d x\right) \\
& -\sum_{j=1}^{3}\left[\int_{\mathbb{R}^{n}}\left(\left|\nabla u_{j, 0}\right|^{2}+u_{j, 0}^{2}\right) d x\right] .
\end{aligned}
$$

Since

$$
F\left(\mu_{1}, 1\right)=0, \quad \frac{\partial F}{\partial t}\left(\mu_{1}, 1\right)>0,
$$

by implicit function theorem, there exists $\delta>0$ such that $t\left(\mu_{1}\right)=1, t\left(\mu_{1}\right) \in C^{1}\left(\mu_{1}-\right.$ $\left.\delta, \mu_{1}+\delta\right)$ and

$$
t^{\prime}\left(\mu_{1}\right)=-\frac{\int_{\mathbb{R}^{n}}\left|u_{1,0}\right|^{4} d x}{2\left(\sum_{j=1}^{3} \mu_{j} \int_{\mathbb{R}^{n}} u_{j, 0}^{4} d x+\int_{\mathbb{R}^{n}} \sum_{i \neq j}^{3} \beta_{i, j} u_{i, 0}^{2} u_{j, 0}^{2} d x\right)} .
$$

By Taylor expansion, we have

$$
t(\mu)=1+t^{\prime}\left(\mu_{1}\right)\left(\mu-\mu_{1}\right)+o\left(\left|\mu-\mu_{1}\right|^{2}\right),
$$

thus,

$$
t^{2}(\mu)=1+2 t^{\prime}\left(\mu_{1}\right)\left(\mu-\mu_{1}\right)+o\left(\left|\mu-\mu_{1}\right|^{2}\right)
$$

Since

$$
\begin{aligned}
& \sum_{j=1}^{3}\left[\int_{\mathbb{R}^{n}}\left(\left|\nabla u_{j, 0}\right|^{2}+u_{j, 0}^{2}\right) d x\right] \\
= & \sum_{j=1}^{3} \mu_{j} \int_{\mathbb{R}^{n}} u_{j, 0}^{4} d x+\int_{\mathbb{R}^{n}} \sum_{i \neq j}^{3} \beta_{i, j} u_{i, 0}^{2} u_{j, 0}^{2} d x \\
= & 4 c\left(\mu_{1}\right),
\end{aligned}
$$

then, by (4.9)-(4.11), we have

$$
\begin{aligned}
& \quad c_{1}(\mu) \leq E_{\mu}\left(t(\mu) u_{1,0}, t(\mu) u_{2,0}, t(\mu) u_{3,0}\right) \\
& =\frac{1}{4} t^{2}(\mu) \sum_{j=1}^{3}\left[\int_{\mathbb{R}^{n}}\left(\left|\nabla u_{j, 0}\right|^{2}+u_{j, 0}^{2}\right) d x\right]=c_{1}\left(\mu_{1}\right) t^{2}(\mu) \\
& =c_{1}\left(\mu_{1}\right)-c_{1}\left(\mu_{1}\right) \frac{\int_{\mathbb{R}^{n}}\left|u_{1,0}\right|^{4} d x}{\left(\sum_{j=1}^{3} \mu_{j} \int_{\mathbb{R}^{n}} u_{j, 0}^{4} d x+\int_{\mathbb{R}^{n}} \sum_{i \neq j}^{3} \beta_{i, j} u_{i, 0}^{2} u_{j, 0}^{2} d x\right)}\left(\mu-\mu_{1}\right) \\
& \quad+o\left(\left|\mu-\mu_{1}\right|^{2}\right) \\
& =c_{1}\left(\mu_{1}\right)-\frac{1}{4} \int_{\mathbb{R}^{n}}\left|u_{1,0}\right|^{4} d x\left(\mu-\mu_{1}\right)+o\left(\left|\mu-\mu_{1}\right|^{2}\right) .
\end{aligned}
$$


Thus

$$
\frac{c_{1}(\mu)-c_{1}\left(\mu_{1}\right)}{\mu-\mu_{1}} \geq-\frac{1}{4} \int_{\mathbb{R}^{n}}\left|u_{1,0}\right|^{4} d x+o\left(\left|\mu-\mu_{1}\right|^{2}\right) \quad \text { as } \mu \nearrow \mu_{1},
$$

so

$$
c_{1}^{\prime}\left(\mu_{1}\right) \geq-\frac{1}{4} \int_{\mathbb{R}^{n}}\left|u_{1,0}\right|^{4} d x
$$

Similarly,

$$
\frac{c_{1}(\mu)-c_{1}\left(\mu_{1}\right)}{\mu-\mu_{1}} \leq-\frac{1}{4} \int_{\mathbb{R}^{n}}\left|u_{1,0}\right|^{4} d x+o\left(\left|\mu-\mu_{1}\right|^{2}\right) \quad \text { as } \mu \searrow \mu_{1}
$$

and

$$
c_{1}^{\prime}\left(\mu_{1}\right) \leq-\frac{1}{4} \int_{\mathbb{R}^{n}}\left|u_{1,0}\right|^{4} d x
$$

Hence

$$
c_{1}^{\prime}\left(\mu_{1}\right)=-\frac{1}{4} \int_{\mathbb{R}^{n}}\left|u_{1,0}\right|^{4} d x .
$$

Since $\left(\eta_{\min } \tau_{1, \min } w, \eta_{\min } \tau_{2, \min } w, \eta_{\min } \tau_{3, \min } w\right)$ is the ground state solution of (2.1), we have

$$
c_{1}^{\prime}\left(\mu_{1}\right)=-\frac{\eta_{\min }^{4} \tau_{1, \min }^{4}}{4} \int_{\mathbb{R}^{n}} w^{4} d x
$$

Thus,

$$
\int_{\mathbb{R}^{n}}\left|u_{1,0}\right|^{4} d x=\eta_{\min }^{4} \tau_{1, \min }^{4} \int_{\mathbb{R}^{n}} w^{4} d x
$$

By the similar arguments as above, that is, by computing $c_{1}^{\prime}\left(\mu_{2}\right), c_{1}^{\prime}\left(\mu_{3}\right)$, we obtain

$$
\int_{\mathbb{R}^{n}}\left|u_{i, 0}\right|^{4} d x=\eta_{\min }^{4} \tau_{i, \min }^{4} \int_{\mathbb{R}^{n}}|w|^{4} d x, \quad i=2,3
$$

Thus, we get (4.3a). Similarly, by computing $c^{\prime}\left(\beta_{i, j}\right)$, we obtain

$$
\int_{\mathbb{R}^{n}}\left|u_{i, 0}\right|^{2}\left|u_{j, 0}\right|^{2} d x=\eta_{\min }^{4} \tau_{i, \min }^{2} \tau_{j, \min }^{2} \int_{\mathbb{R}^{n}}|w|^{4} d x \quad \text { for } i<j=1,2,3
$$

Thus, by (4.3a), (4.3b), we have

$$
\int_{\mathbb{R}^{n}}\left|u_{i, 0}\right|^{2}\left|u_{j, 0}\right|^{2} d x=\tau_{i, \min }^{-2} \tau_{j, \min }^{2} \int_{\mathbb{R}^{n}}\left|u_{i, 0}\right|^{4} d x \quad \text { for } i<j=1,2,3
$$

Next, we prove

$$
\left(u_{1,0}, u_{2,0}, u_{3,0}\right)=\left(\eta_{\min } \tau_{1, \min } w, \eta_{\min } \tau_{2, \min } w, \eta_{\min } \tau_{3, \min } w\right) .
$$


Since $\left(\eta_{\min } \tau_{1, \min } w, \eta_{\min } \tau_{2, \min } w, \eta_{\min } \tau_{3, \min } w\right)$ is a ground state solution of (2.1), we have

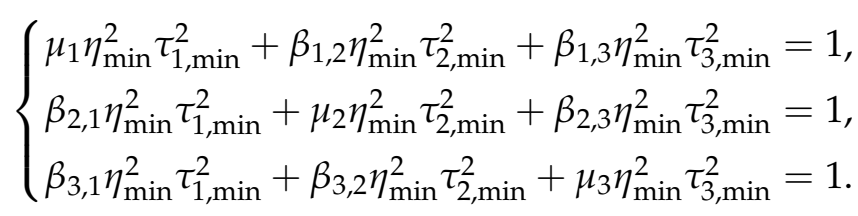

Let

$$
\left(u_{1}, u_{2}, u_{3}\right)=\left(\frac{u_{1,0}}{\eta_{\min } \tau_{1, \min }}, \frac{u_{2,0}}{\eta_{\min } \tau_{2, \min }}, \frac{u_{3,0}}{\eta_{\min } \tau_{3, \min }}\right),
$$

then, by (4.12) and (4.13), we have

$$
\begin{aligned}
\int_{\mathbb{R}^{n}}\left(\left|\nabla u_{i}\right|^{2}+u_{i}^{2}\right) d x & =\frac{1}{\eta_{\min }^{2} \tau_{i, \min }^{2}} \int_{\mathbb{R}^{n}}\left(\left|\nabla u_{i, 0}\right|^{2}+u_{i, 0}^{2}\right) d x \\
& =\frac{1}{\eta_{\min }^{2} \tau_{i, \min }^{2}} \int_{\mathbb{R}^{n}}\left(\mu_{i} u_{i, 0}^{4}+\sum_{i \neq j} \beta_{i, j} u_{i, 0}^{2} u_{j, 0}^{2}\right) d x \\
& =\frac{1}{\eta_{\min }^{2} \tau_{i, \min }^{2}}\left(\mu_{i}+\sum_{i \neq j} \beta_{i, j} \tau_{i, \min }^{-2} \tau_{j, \min }^{2}\right) \int_{\mathbb{R}^{n}}\left|u_{i, 0}\right|^{4} d x \\
& =\frac{1}{\eta_{\min }^{4} \tau_{i, \min }^{4}} \int_{\mathbb{R}^{n}}\left|u_{i, 0}\right|^{4} d x \\
& =\int_{\mathbb{R}^{n}}\left|u_{i}\right|^{4} d x, \quad i=1,2,3 .
\end{aligned}
$$

Hence,

$$
\int_{\mathbb{R}^{n}}\left(\left|\nabla u_{i}\right|^{2}+u_{i}^{2}\right) d x \geq \int_{\mathbb{R}^{n}}\left(|\nabla w|^{2}+w^{2}\right) d x, \quad i=1,2,3 .
$$

Since $\left(u_{1,0}, u_{2,0}, u_{3,0}\right)$ and $\left(\eta_{\min } \tau_{1, \min } w, \eta_{\min } \tau_{2, \min } w, \eta_{\min } \tau_{3, \min } w\right)$ are both the ground state solution of (2.1), we obtain

$$
\begin{aligned}
& \frac{1}{4} \sum_{i}^{3} \eta_{\min }^{2} \tau_{i, \min }^{2} \int_{\mathbb{R}^{n}}\left(|\nabla w|^{2}+w^{2}\right) d x=\frac{1}{4} \sum_{i}^{3} \int_{\mathbb{R}^{n}}\left(\left|\nabla u_{i, 0}\right|^{2}+u_{i, 0}^{2}\right) d x \\
= & \frac{1}{4} \sum_{i}^{3} \eta_{\min }^{2} \tau_{i, \min }^{2} \int_{\mathbb{R}^{n}}\left(\left|\nabla u_{i}\right|^{2}+u_{i}^{2}\right) d x \geq \frac{1}{4} \sum_{i}^{3} \eta_{\min }^{2} \tau_{i, \min }^{2} \int_{\mathbb{R}^{n}}\left(|\nabla w|^{2}+w^{2}\right) d x,
\end{aligned}
$$

which implies that

$$
\int_{\mathbb{R}^{n}}\left(\left|\nabla u_{i}\right|^{2}+u_{i}^{2}\right) d x=\int_{\mathbb{R}^{n}}\left(|\nabla w|^{2}+w^{2}\right) d x, \quad i=1,2,3 .
$$

So, $u_{i}, i=1,2,3$ are positive ground state solutions of (1.5). Since $\left(u_{1,0}, u_{2,0}, u_{3,0}\right)$ satisfies (2.1) and

$$
\mu_{1} \eta_{\min }^{2} \tau_{1, \min }^{2}+\beta_{21} \eta_{\min }^{2} \tau_{2, \min }^{2}+\beta_{31} \eta_{\min }^{2} \tau_{3, \min }^{2}=1,
$$


we have

$$
-\Delta u_{1}+u_{1}=\mu_{1} \eta_{\min }^{2} \tau_{1, \min }^{2} u_{1}^{3}+\beta_{2,1} \eta_{\min }^{2} \tau_{2, \min }^{2} u_{2}^{2} u_{1}+\beta_{3,1} \eta_{\min }^{2} \tau_{3, \min }^{2} u_{3}^{2} u_{1}=u_{1}^{3} .
$$

So

$$
u_{1}^{3}=u_{i}^{2} u_{1} \quad \text { and } \quad u_{i}=u_{1}, \quad i=2,3 .
$$

Since (1.5) has a unique positive ground state solution $w$, thus, we have

$$
\left(u_{1,0}, u_{2,0}, u_{3,0}\right)=\left(\eta_{\min } \tau_{1, \min } w, \eta_{\min } \tau_{2, \min } w, \eta_{\min } \tau_{3, \min }\right)
$$

The proof is thus completed.

The proof of second part of Theorem 2.4 is similar to the proof second part of Theorem 2.1. We choose auxiliary function as following

$$
g^{\mu}\left(\mu, \tau_{1}, \tau_{2}, \cdots, \tau_{N}\right)=\frac{\sum_{i=1}^{N} \tau_{i}^{2}}{\left(\mu \tau_{1}^{4}+\sum_{i=2}^{N} \mu_{i} \tau_{i}^{4}+2 \sum_{i, j=1, i<j}^{N} \beta_{i, j} \tau_{i}^{2} \tau_{j}^{2}\right)^{\frac{1}{2}}} .
$$

So

$$
g_{\tau_{i}}^{\mu}\left(\mu, \tau_{1}, \tau_{2}, \cdots, \tau_{N}\right)=\frac{2 \tau_{i} H_{i}^{\mu}\left(\mu, \tau_{1}, \tau_{2}, \cdots, \tau_{N}\right)}{\left(\mu \tau_{1}^{4}+\sum_{i=2}^{N} \mu_{i} \tau_{i}^{4}+2 \sum_{i, j=1, i<j}^{N} \beta_{i, j} \tau_{i}^{2} \tau_{j}^{2}\right)^{\frac{3}{2}}}=0
$$

where

$$
\begin{aligned}
& H_{i}^{\mu}\left(\mu, \tau_{1}, \tau_{2}, \cdots, \tau_{N}\right) \\
= & \mu \tau_{1}^{4}+\sum_{i=2}^{N} \mu_{i} \tau_{i}^{4}+2 \sum_{i, j=1, i<j}^{N} \beta_{i, j} \tau_{i}^{2} \tau_{j}^{2}-\left(\tau_{1}^{2}+\tau_{2}^{2}+\cdots+\tau_{N}^{2}\right)\left(\sum_{j=1}^{N} \beta_{i, j} \tau_{j}^{2}\right) \\
= & \tau_{1}^{2}\left(\left(\mu-\beta_{i, 1}\right) \tau_{1}^{2}+\sum_{j=2}^{N}\left(\beta_{1, j}-\beta_{i, j}\right) \tau_{j}^{2}\right)+\tau_{2}^{2}\left(\sum_{j=1}^{N}\left(\beta_{2, j}-\beta_{i, j}\right) \tau_{j}^{2}\right) \cdots \\
& +\tau_{i-1}^{2}\left(\sum_{j=1}^{N}\left(\beta_{i-1, j}-\beta_{i, j}\right) \tau_{j}^{2}\right)+\tau_{i+1}^{2}\left[\sum_{j=1}^{N}\left(\beta_{i+1, j}-\beta_{i, j}\right) \tau_{j}^{2}\right] \cdots \\
& +\tau_{N-1}^{2}\left(\sum_{j=1}^{N}\left(\beta_{N-1, j}-\beta_{i, j}\right) \tau_{j}^{2}\right)+\tau_{N}^{2}\left(\sum_{j=1}^{N}\left(\beta_{N, j}-\beta_{i, j}\right) \tau_{j}^{2}\right) .
\end{aligned}
$$


Thus $H_{i}^{\mu}\left(\mu, \tau_{1}, \tau_{2}, \cdots, \tau_{N}\right)=0, i=1, \cdots, N$, which implies that

$$
\left\{\begin{array}{l}
\left(\mu-\beta_{i, 1}\right) \tau_{1}^{2}+\sum_{j=2}^{N}\left(\beta_{1, j}-\beta_{i, j}\right) \tau_{j}^{2}=0 \\
\sum_{j=1}^{N}\left(\beta_{2, j}-\beta_{i, j}\right) \tau_{j}^{2}=0 \\
\vdots \\
\sum_{j=1}^{N}\left(\beta_{i-1, j}-\beta_{i, j}\right) \tau_{j}^{2}=0 \\
\sum_{j=1}^{N}\left(\beta_{i+1, j}-\beta_{i, j}\right) \tau_{j}^{2}-0, \\
\vdots \\
\sum_{j=1}^{N}\left(\beta_{N-1, j}-\beta_{i, j}\right) \tau_{j}^{2}=0 \\
\sum_{j=1}^{N}\left(\beta_{N, j}-\beta_{i, j}\right) \tau_{j}^{2}=0 .
\end{array}\right.
$$

The other part of the proof is similar as the proof of the second part of Theorem 2.1.

\section{Proof of Theorem 2.2 and Theorem 2.5}

Proof of Theorem 2.2 and Theorem 2.5. We follow some ideas from [10] and recent work [29], where two-coupled system was considered. Indeed, by Lemma 1 and Lemma 2 in [16], $w$ is the unique function attaining $S$. Thus,

$$
\int_{\mathbb{R}^{n}}\left(|\nabla w|^{2}+w^{2}\right) d x=\int_{\mathbb{R}^{n}} w^{4} d x=S^{2} .
$$

On the one hand, if $d_{1}, d_{2}, d_{3}$ satisfy (2.5), it is easy to see that

$$
\left(d_{1} w, d_{2} w, d_{3} w\right)
$$

satisfies (2.1) and belongs to $\mathbf{M}_{3}$. So

$$
c_{3} \leq E\left(d_{1} w, d_{2} w, d_{3} w\right)=\frac{1}{4} \sum_{j=1}^{3} d_{j}^{2} \int_{\mathbb{R}^{n}}\left(|\nabla w|^{2}+w^{2}\right) d x=\frac{1}{4} \sum_{j=1}^{3} d_{j}^{2} s^{2} .
$$

On the other hand, let $\left(u_{1, n}, u_{2, n}, u_{3, n}\right) \in \mathbf{M}_{3}$ be a minimizing sequence for $c_{3}$, that is

$$
E\left(u_{1, n}, u_{2, n}, u_{3, n}\right) \rightarrow c_{3} \text { as } n \rightarrow+\infty \text {. }
$$


Define

$$
q_{j, n}=\left(\int_{\mathbb{R}^{n}} u_{j, n}^{4} d x\right)^{\frac{1}{2}}, \quad j=1,2,3,
$$

then by the definition of $S$ (see (3.2a)) and Hölder inequality, we have

$$
\begin{aligned}
& S\left(\int_{\mathbb{R}^{n}} u_{1, n}^{4} d x\right)^{\frac{1}{2}} \leq \int_{\mathbb{R}^{n}}\left(\left|\nabla u_{1, n}\right|^{2}+u_{1, n}^{2}\right) d x \\
= & \mu_{1} \int_{\mathbb{R}^{n}} u_{1, n}^{4} d x+\sum_{j \neq 1}^{3} \beta_{1, j} \int_{\mathbb{R}^{n}} u_{1, n}^{2} u_{j, n}^{2} d x \\
\leq & \mu_{1} \int_{\mathbb{R}^{n}} u_{1, n}^{4} d x+\sum_{j \neq 1}^{3} \beta_{1, j}\left(\int_{\mathbb{R}^{n}} u_{1, n}^{4} d x\right)^{\frac{1}{2}}\left(\int_{\mathbb{R}^{n}} u_{j, n}^{4} d x\right)^{\frac{1}{2}} .
\end{aligned}
$$

Thus

$$
S \leq \mu_{1} q_{1, n}+\beta_{1,2} q_{2, n}+\beta_{1,3} q_{3, n}
$$

Similarly, we have

$$
\begin{aligned}
& S \leq \mu_{2} q_{2, n}+\beta_{2,1} q_{1, n}+\beta_{2,3} q_{3, n}, \\
& S \leq \mu_{3} q_{3, n}+\beta_{3,1} q_{1, n}+\beta_{3,2} q_{2, n} .
\end{aligned}
$$

Since

$$
E\left(u_{1, n}, u_{2, n}, u_{3, n}\right)=\frac{1}{4} \sum_{j=1}^{3} \int_{\mathbb{R}^{n}}\left(\left|\nabla u_{j, n}\right|^{2}+u_{j, n}^{2}\right) d x
$$

we have

$$
\begin{aligned}
& S \sum_{j=1}^{3} q_{j, n} \leq \sum_{j=1}^{3} \int_{\mathbb{R}^{n}}\left(\left|\nabla u_{j, n}\right|^{2}+u_{j, n}^{2}\right) d x \\
= & 4 E\left(u_{1, n}, u_{2, n}, u_{3, n}\right)+o(1) \\
= & 4 c_{3}+o(1) \leq \sum_{j=1}^{3} d_{j}^{2} S^{2}+o(1) .
\end{aligned}
$$

Thus

$$
\sum_{j=1}^{3} q_{j, n} \leq \sum_{j=1}^{3} d_{j}^{2} S+o(1) .
$$

By (2.5), (5.2) to (5.4), we obtain

$$
\left\{\begin{array}{l}
\mu_{1}\left(q_{1, n}-d_{1}^{2} S\right)+\beta_{1,2}\left(q_{2, n}-d_{2}^{2} S\right)+\beta_{1,3}\left(q_{3, n}-d_{3}^{2} S\right) \geq 0 \\
\beta_{2,1}\left(q_{1, n}-d_{1}^{2} S\right)+\mu_{2}\left(q_{2, n}-d_{2}^{2} S\right)+\beta_{2,3}\left(q_{3, n}-d_{3}^{2} S\right) \geq 0 \\
\beta_{3,1}\left(q_{1, n}-d_{1}^{2} S\right)+\beta_{3,2}\left(q_{2, n}-d_{2}^{2} S\right)+\mu_{3}\left(q_{3, n}-d_{3}^{2} S\right) \geq 0 \\
\left(q_{1, n}-d_{1}^{2} S\right)+\left(q_{2, n}-d_{2}^{2} S\right)+\left(q_{3, n}-d_{3}^{2} S\right) \leq o(1) .
\end{array}\right.
$$


We claim

$$
\left(q_{1, n}-d_{1}^{2} S\right)+\left(q_{2, n}-d_{2}^{2} S\right)+\left(q_{3, n}-d_{3}^{2} S\right) \rightarrow 0 \text { as } n \rightarrow+\infty .
$$

Indeed, from (5.5), we have

$$
\mathcal{B} \mathbf{X} \geq \mathbf{0}
$$

where

$$
\mathcal{B}=\left(\begin{array}{lll}
\mu_{1}, & \beta_{12}, & \beta_{13} \\
\beta_{21}, & \mu_{2}, & \beta_{23} \\
\beta_{31}, & \beta_{32}, & \mu_{3}
\end{array}\right), \quad \mathbf{X}=\left(\begin{array}{l}
q_{1, n}-d_{1}^{2} S \\
q_{2, n}-d_{2}^{2} S \\
q_{3, n}-d_{3}^{2} S
\end{array}\right), \quad \mathbf{0}=\left(\begin{array}{l}
0 \\
0 \\
0
\end{array}\right) .
$$

Next, we show

$$
\left(q_{1, n}-d_{1}^{2} S\right)+\left(q_{2, n}-d_{2}^{2} S\right)+\left(q_{3, n}-d_{3}^{2} S\right) \geq 0 .
$$

Since $\mathcal{B}^{-}$is the invertible matrix of $\mathcal{B}$, we let

$$
\mathcal{B X}=\mathbf{C} \geq \mathbf{0} \Rightarrow \mathbf{X}=\mathcal{B}^{-} \mathbf{C},
$$

where

$$
\mathcal{B}^{-}=\left(\begin{array}{lll}
\beta^{1,1}, & \beta^{1,2}, & \beta^{1,3} \\
\beta^{2,1}, & \beta^{2,2}, & \beta^{2,3} \\
\beta^{3,1}, & \beta^{3,2}, & \beta^{3,3}
\end{array}\right), \quad \mathbf{C}=\left(\begin{array}{l}
C_{1} \\
C_{2} \\
C_{3}
\end{array}\right)
$$

then, if $\sum_{i=1}^{3} \beta^{i, k} \geq 0$ for all $k=1,2,3$, we have

$$
\sum_{i=1}^{3}\left(q_{i, n}-d_{i}^{2} S\right)=\sum_{i=1}^{3} \sum_{k=1}^{3} \beta^{i, k} C_{k}=\left(\sum_{k=1}^{3} C_{k}\right)\left(\sum_{i=1}^{3} \beta^{i, k}\right) \geq 0 .
$$

By (5.5) and (5.6), we have

$$
\left(q_{1, n}-d_{1}^{2} S\right)+\left(q_{2, n}-d_{2}^{2} S\right)+\left(q_{3, n}-d_{3}^{2} S\right) \rightarrow 0 \text { as } n \rightarrow+\infty .
$$

Thus,

$$
\sum_{j=1}^{3} q_{j, n} \rightarrow \sum_{j=1}^{3} d_{j}^{2} S \quad \text { as } n \rightarrow+\infty
$$

So

$$
c_{3}=\lim _{n \rightarrow+\infty} E\left(u_{1, n}, u_{2, n}, u_{3, n}\right) \geq \frac{1}{4} S \sum_{j=1}^{3} q_{j, n}=\frac{1}{4} \sum_{j=1}^{3} d_{j}^{2} S^{2} .
$$

Combining (5.1) with (5.7), we have

$$
c_{3}=\frac{1}{4} \sum_{j=1}^{3} d_{j}^{2} S^{2}=E\left(d_{1} w, d_{2} w, d_{3} w\right) .
$$


Thus, $\left(d_{1} w, d_{2} w, d_{3} w\right)$ is a positive ground state solution of (2.1). The proof of Theorem 2.5 are similar to above proof.

To proof the uniqueness of positive ground state solution of (2.1), we show that if $\left(u_{1,0}, u_{2,0}, u_{3,0}\right)$ be any ground state solution of $(2.1)$, then

$$
\left(u_{1,0}, u_{2,0}, u_{3,0}\right)=\left(d_{1} w, d_{2} w, d_{3} w\right)
$$

Let $\left(u_{1,0}, u_{2,0}, u_{3,0}\right)$ be any ground state solution of (2.1), then by strong maximum principle, we have $u_{j, 0}>0, j=1,2,3$. We claim

$$
\begin{array}{ll}
\int_{\mathbb{R}^{n}} u_{i, 0}^{4} d x=d_{i}^{4} \int_{\mathbb{R}^{n}} w^{4} d x & \text { for } i=1,2,3, \\
\int_{\mathbb{R}^{n}} u_{i, 0}^{2} u_{j, 0}^{2} d x=d_{i}^{2} d_{j}^{2} \int_{\mathbb{R}^{n}} w^{4} d x & \text { for } i, j=1,2,3 .
\end{array}
$$

To proof the claim, we consider the following system, where $\mu_{1}$ is replaced by $\mu$ in system (2.1).

$$
\begin{cases}-\Delta u_{1}+u_{1}=\mu u_{1}^{3}+\beta_{1,2} u_{2}^{2} u_{1}+\beta_{1,3} u_{3}^{2} u_{1} & \text { in } \mathbb{R}^{n} \\ -\Delta u_{2}+u_{2}=\mu_{2} u_{2}^{3}+\beta_{2,1} u_{1}^{2} u_{2}+\beta_{2,3} u_{3}^{2} u_{2} & \text { in } \mathbb{R}^{n} \\ -\Delta u_{3}+u_{3}=\mu_{3} u_{3}^{3}+\beta_{3,1} u_{1}^{2} u_{3}+\beta_{3,2} u_{2}^{2} u_{3} & \text { in } \mathbb{R}^{n} \\ u_{i}>0 \quad \text { in } \mathbb{R}^{n}, \quad u_{i}(x) \rightarrow 0 & \text { as }|x| \rightarrow+\infty, \quad i=1,2,3 .\end{cases}
$$

The corresponding energy functional is given by

$$
\begin{gathered}
E_{\mu}\left(\mu, u_{1}, u_{2}, u_{3}\right)=\sum_{j=1}^{3} \frac{1}{2} \int_{\mathbb{R}^{n}}\left(\left|\nabla u_{j}\right|^{2}+u_{j}^{2}\right) d x-\frac{1}{4} \sum_{i \neq j}^{3} \beta_{i, j} u_{i}^{2} u_{j}^{2} d x \\
-\mu \int_{\mathbb{R}^{n}} u_{1}^{4} d x-\sum_{j=2}^{3} \frac{1}{4} \mu_{j} \int_{\mathbb{R}^{n}} u_{j}^{4} d x .
\end{gathered}
$$

It is easy to see

$$
c_{1}(\mu)=\inf _{\left(u_{1}, u_{2}, u_{3}\right) \in \mathcal{H} \backslash\{(0,0,0)\}} \max _{t>0} E_{\mu}\left(\mu, t u_{1}, t u_{2}, t u_{3}\right) .
$$

The next steps are same as the proof of Theorem 2.1 and Theorem 2.4. Thus, we have proof

$$
\left(u_{1,0}, u_{2,0}, u_{3,0}\right)=\left(d_{1} w, d_{2} w, d_{3} w\right)
$$

Thus, we complete the proof. 


\section{Proof of Theorem 2.3}

Proof of Theorem 2.3. Step 1 . When $\frac{d_{2}}{d_{1}}=m$, then $\left(d_{1} w, d_{2} w, d_{3} w\right)$ is a positive ground state solution of (2.1). On the one hand, if $d_{1}, d_{3}$ satisfy following equation,

$$
\left\{\begin{array}{l}
\left(\mu_{1}+\beta_{1,2} m^{2}\right) d_{1}^{2}+\beta_{1,3} d_{3}^{2}=1, \\
\left(\mu_{2} m^{2}+\beta_{2,1}\right) d_{1}^{2}+\beta_{2,3} d_{3}^{2}=1 \\
\left(\beta_{3,1}+\beta_{3,2} m^{2}\right) d_{1}^{2}+\mu_{3} d_{3}^{2}=1 .
\end{array}\right.
$$

So

$$
\left\{\begin{array}{l}
\left(\mu_{1}+\mu_{2} k^{4}+2 \beta_{1,2} m^{2}\right) d_{1}^{2}+\left(\beta_{1,3}+\beta_{23} m^{2}\right) d_{3}^{2}=1+m^{2}, \\
\left(\beta_{3,1}+\beta_{3,2} m^{2}\right) d_{1}^{2}+\mu_{3} d_{3}^{2}=1,
\end{array}\right.
$$

it is easy to see that $\left(d_{1} w, m d_{1} w, d_{3} w\right)$ satisfies (2.1) and belongs to $\mathbf{M}_{2}$. So

$$
\begin{aligned}
c_{2} & \leq E\left(d_{1} w, m d_{1} w, d_{3} w\right) \\
& =\frac{1}{4}\left(\left(1+m^{2}\right) d_{1}^{2}+d_{3}^{2}\right) \int_{\mathbb{R}^{n}}\left(|\nabla w|^{2}+w^{2}\right) d x \\
& =\frac{1}{4}\left(\left(1+m^{2}\right) d_{1}^{2}+d_{3}^{2}\right) S^{2} .
\end{aligned}
$$

On the other hand, let $\left(u_{12, n}, m u_{12, n}, u_{3, n}\right) \in \mathbf{M}_{2}$ be a minimizing sequence for $c_{2}$, that is

$$
E\left(u_{12, n}, m u_{12, n}, u_{3, n}\right) \rightarrow c_{2} \text { as } n \rightarrow+\infty \text {. }
$$

Define

$$
q_{12, n}=\left(\int_{\mathbb{R}^{n}} u_{12, n}^{4} d x\right)^{\frac{1}{2}}, \quad q_{3, n}=\left(\int_{\mathbb{R}^{n}} u_{3, n}^{4} d x\right)^{\frac{1}{2}},
$$

then by the similar arguments as (5.2), we have

$$
\begin{aligned}
& S \leq \mu_{3} q_{3, n}+\left(\beta_{3,1}+\beta_{3,2} m^{2}\right) q_{12, n} \\
& \left(1+m^{2}\right) S \leq\left(\mu_{1}+\mu_{2} k^{4}+2 \beta_{1,2} m^{2}\right) q_{12, n}+\left(\beta_{1,3}+\beta_{2,3} m^{2}\right) q_{3, n} .
\end{aligned}
$$

Since

$$
\begin{aligned}
& S\left(\left(1+m^{2}\right) q_{12, n}+q_{3, n}\right) \\
\leq & \left(1+m^{2}\right) \int_{\mathbb{R}^{n}}\left(\left|\nabla u_{12, n}\right|^{2}+u_{12, n}^{2}\right) d x+\int_{\mathbb{R}^{n}}\left(\left|\nabla u_{3, n}\right|^{2}+u_{3, n}^{2}\right) d x \\
= & 4 E\left(u_{12, n}, m u_{12, n}, u_{3, n}\right)+o(1) \\
= & 4 c_{2}+o(1) \\
\leq & \left(\left(1+m^{2}\right) d_{1}^{2}+d_{3}^{2}\right) S^{2}+o(1) .
\end{aligned}
$$


So

$$
\left(1+m^{2}\right)\left(q_{12, n}-d_{1}^{2} S\right)+\left(q_{3, n}-d_{3}^{2} S\right) \leq o(1) .
$$

Thus, by (6.1), (6.3a), (6.3b) and (6.4), we have

$$
\left\{\begin{array}{l}
\left(1+m^{2}\right)\left(q_{12, n}-d_{1}^{2} S\right)+\left(q_{3, n}-d_{3}^{2} S\right) \leq o(1) \\
\left(\mu_{1}+\mu_{2} k^{4}+2 \beta_{1,2} m^{2}\right)\left(q_{12, n}-d_{1}^{2} S\right)+\left(\beta_{1,3}+\beta_{2,3} m^{2}\right)\left(q_{3, n}-d_{3}^{2} S\right) \geq 0 \\
\left(\beta_{3,1}+\beta_{3,2} m^{2}\right)\left(q_{12, n}-d_{1}^{2} S\right)+\mu_{3}\left(q_{3, n}-d_{3}^{2} S\right) \geq 0 .
\end{array}\right.
$$

Next, we claim

$$
\left(1+m^{2}\right)\left(q_{12, n}-d_{1}^{2} S\right)+\left(q_{3, n}-d_{3}^{2} S\right) \rightarrow 0 \quad \text { as } n \rightarrow+\infty .
$$

From (6.5), we have

$$
\mathcal{D} \mathbf{X} \geq \mathbf{0}
$$

where

$$
\mathcal{D}=\left(\begin{array}{cc}
\mu_{1}+\mu_{2} k^{4}+2 \beta_{1,2} m^{2}, & \beta_{1,3}+\beta_{2,3} m^{2} \\
\beta_{3,1}+\beta_{3,2} m^{2}, & \mu_{3}
\end{array}\right), \quad \mathbf{X}=\left(\begin{array}{c}
q_{12, n}-d_{1}^{2} S \\
q_{3, n}-d_{3}^{2} S
\end{array}\right), \quad \mathbf{0}=\left(\begin{array}{l}
0 \\
0
\end{array}\right) .
$$

Next, we show

$$
\left(1+m^{2}\right)\left(q_{12, n}-d_{1}^{2} S\right)+\left(q_{3, n}-d_{3}^{2} S\right) \geq 0
$$

Since $\mathbf{D}^{-}$is the invertible matrix of $\mathcal{D}$, we let

$$
\mathcal{D} \mathbf{X}=\mathbf{C} \geq \mathbf{0} \Rightarrow \mathbf{X}=\mathbf{D}^{-} \mathbf{C},
$$

where

$$
\mathbf{D}^{-}=\left(\begin{array}{ll}
D^{11}, & D^{12} \\
D^{21}, & D^{22}
\end{array}\right), \quad \mathbf{C}=\left(\begin{array}{l}
C_{1} \\
C_{2}
\end{array}\right)
$$

If we let

$$
\left\{\begin{array}{l}
\left(1+m^{2}\right) D^{11}+D^{21} \geq 0 \\
\left(1+m^{2}\right) D^{12}+D^{22} \geq 0
\end{array}\right.
$$

then

$$
\begin{aligned}
& \left(1+m^{2}\right)\left(q_{12, n}-d_{1}^{2} S\right)+q_{3, n}-d_{3}^{2} S \\
= & \left(\left(1+m^{2}\right) D^{11}+D^{21}\right) C_{1}+\left(\left(1+m^{2}\right) D^{12}+D^{22}\right) C_{2} \\
\geq & 0 .
\end{aligned}
$$

So,

$$
\left(1+m^{2}\right)\left(q_{12, n}-d_{1}^{2} S\right)+q_{3, n}-d_{3}^{2} S \rightarrow 0 \quad \text { as } n \rightarrow+\infty .
$$


Thus,

$$
\left(1+m^{2}\right) q_{12, n}+q_{3, n} \rightarrow\left(1+m^{2}\right) d_{1}^{2} S+d_{3}^{2} S \quad \text { as } n \rightarrow+\infty .
$$

So,

$$
\begin{aligned}
c_{2} & =\lim _{n \rightarrow+\infty} E\left(u_{12, n}, m u_{12, n}, u_{3, n}\right) \\
& \geq \frac{1}{4} S\left(\left(1+m^{2}\right) q_{12, n}+q_{3, n}\right) \\
& =\frac{1}{4}\left(\left(1+m^{2}\right) d_{1}^{2}+d_{3}^{2}\right) S^{2} .
\end{aligned}
$$

Thus,

$$
c_{2}=\frac{1}{4}\left(\left(1+m^{2}\right) d_{1}^{2}+d_{3}^{2}\right) S^{2}
$$

So when

$$
\frac{d_{2}}{d_{1}}=m
$$

$\left(d_{1} w, d_{2} w, d_{3} w\right)$ is a positive ground state solution of (2.1).

Step 2 . We show that if $\left(u_{12,0}, m u_{12,0}, u_{3,0}\right)$ be any ground state solution of $(2.1)$, then

$$
\left(u_{12,0}, m u_{12,0}, u_{3,0}\right)=\left(d_{1} w, m d_{1} w, d_{3} w\right) .
$$

The next steps are similar as we proof Theorem 2.1, for readers conveniences, we give the details here.

Let $\left(u_{12,0}, m u_{12,0}, u_{3,0}\right)$ be any ground state solution of $(2.1)$, then by strong maximum principle, we have $u_{12,0}>0, u_{3,0}>0$. We claim

$$
\begin{aligned}
& \int_{\mathbb{R}^{n}} u_{12,0}^{4} d x=d_{1}^{4} \int_{\mathbb{R}^{n}} w^{4} d x, \\
& \int_{\mathbb{R}^{n}} u_{3,0}^{4} d x=d_{3}^{4} \int_{\mathbb{R}^{n}} w^{4} d x, \\
& \int_{\mathbb{R}^{n}} u_{12,0}^{2} u_{3,0}^{2} d x=d_{1}^{2} d_{3}^{2} \int_{\mathbb{R}^{n}} w^{4} d x .
\end{aligned}
$$

To proof the claim, we consider the following system, where $\mu_{1}$ is replaced by $\mu$ in system (2.1).

$$
\begin{cases}-\Delta u_{1}+u_{1}=\mu u_{1}^{3}+\beta_{1,2} u_{2}^{2} u_{1}+\beta_{1,3} u_{3}^{2} u_{1} & \text { in } \mathbb{R}^{n} \\ -\Delta u_{2}+u_{2}=\mu_{2} u_{2}^{3}+\beta_{2,1} u_{1}^{2} u_{2}+\beta_{2,3} u_{3}^{2} u_{2} & \text { in } \mathbb{R}^{n} \\ -\Delta u_{3}+u_{3}=\mu_{3} u_{3}^{3}+\beta_{3,1} u_{1}^{2} u_{3}+\beta_{3,2} u_{2}^{2} u_{3} & \text { in } \mathbb{R}^{n} \\ u_{i}>0 \quad \text { in } \mathbb{R}^{n}, \quad u_{i}(x) \rightarrow 0 & \text { as }|x| \rightarrow+\infty, \quad i=1,2,3 .\end{cases}
$$


The corresponding energy functional is given by

$$
\begin{gathered}
E_{\mu}\left(\mu, u_{1}, u_{2}, u_{3}\right)=\sum_{j=1}^{3} \frac{1}{2} \int_{\mathbb{R}^{n}}\left(\left|\nabla u_{j}\right|^{2}+u_{j}^{2}\right) d x-\frac{1}{4} \sum_{i \neq j}^{3} \beta_{i, j} u_{i}^{2} u_{j}^{2} d x \\
-\mu \int_{\mathbb{R}^{n}} u_{1}^{4} d x-\sum_{j=2}^{3} \frac{1}{4} \mu_{j} \int_{\mathbb{R}^{n}} u_{j}^{4} d x .
\end{gathered}
$$

It is easy to see

$$
c_{2}(\mu)=\inf _{\left(u_{1}, m u_{1}, u_{3}\right) \in \mathcal{H} \backslash\{(0,0,0)\}} \max _{t>0} E_{\mu}\left(\mu, t u_{1}, t m u_{1}, t u_{3}\right) .
$$

Thus, there exists $t(\mu)>0$ such that

$$
E_{\mu}\left(\mu, t(\mu) u_{12,0}, t(\mu) m u_{12,0}, t(\mu) u_{3,0}\right)=\max _{t>0} E_{\mu}\left(\mu, t u_{1}, t m u_{1}, t u_{3}\right),
$$

where $t(\mu)>0$ satisfies $F(\mu, t(\mu))=0$ and $F(\mu, t)$ is defined as following

$$
\begin{aligned}
F(\mu, t)=t^{2} & \left(\mu \int_{\mathbb{R}^{n}} u_{12,0}^{4} d x+\mu_{2} m^{4} \int_{\mathbb{R}^{n}} u_{12,0}^{4} d x+\mu_{3} \int_{\mathbb{R}^{n}} u_{3,0}^{4} d x\right. \\
& \left.+\int_{\mathbb{R}^{n}} 2 \beta_{1,2} m^{2} u_{12,0}^{4} d x+\int_{\mathbb{R}^{n}}\left(2 \beta_{1,3}+2 \beta_{2,3} m^{2}\right) u_{12,0}^{2} u_{3,0}^{2} d x\right) \\
& -\left[\left(1+m^{2}\right) \int_{\mathbb{R}^{n}}\left(\left|\nabla u_{12,0}\right|^{2}+u_{12,0}^{2}\right) d x+\int_{\mathbb{R}^{n}}\left(\left|\nabla u_{3,0}\right|^{2}+u_{3,0}^{2}\right) d x\right],
\end{aligned}
$$

then

$$
F\left(\mu_{1}, 1\right)=0, \quad \frac{\partial F(\mu, t)}{\partial t}\left(\mu_{1}, 1\right)>0,
$$

by implicit function theorem, there exists $\delta>0$ such that $t\left(\mu_{1}\right)=1, t\left(\mu_{1}\right) \in C^{1}\left(\mu_{1}-\right.$ $\left.\delta, \mu_{1}+\delta\right)$ and

$$
\begin{aligned}
& t^{\prime}\left(\mu_{1}\right) \\
= & -\frac{\int_{\mathbb{R}^{n}}\left|u_{12,0}\right|^{4} d x}{2\left(\left(\mu_{1}+\mu_{2} m^{4}+2 \beta_{1,2} m^{2}\right) \int_{\mathbb{R}^{n}} u_{12,0}^{4} d x+\int_{\mathbb{R}^{n}}\left(2 \beta_{1,3}+2 \beta_{2,3} m^{2}\right) u_{12,0}^{2} u_{3,0}^{2} d x+\mu_{3} \int_{\mathbb{R}^{n}} u_{3,0}^{4} d x\right)} .
\end{aligned}
$$

By Taylor expansion, we have

$$
t(\mu)=1+t^{\prime}\left(\mu_{1}\right)\left(\mu-\mu_{1}\right)+o\left(\left|\mu-\mu_{1}\right|^{2}\right)
$$

thus,

$$
t^{2}(\mu)=1+2 t^{\prime}\left(\mu_{1}\right)\left(\mu-\mu_{1}\right)+o\left(\left|\mu-\mu_{1}\right|^{2}\right)
$$


Since

$$
\begin{aligned}
& 4 c_{2}\left(\mu_{1}\right)=\left(1+m^{2}\right) \int_{\mathbb{R}^{n}}\left(\left|\nabla u_{12,0}\right|^{2}+u_{12,0}^{2}\right) d x+\int_{\mathbb{R}^{n}}\left(\left|\nabla u_{3,0}\right|^{2}+u_{3,0}^{2}\right) d x \\
& =\left(\left(\mu_{1}+\mu_{2} m^{4}+2 \beta_{1,2} m^{2}\right) \int_{\mathbb{R}^{n}} u_{12,0}^{4} d x+\int_{\mathbb{R}^{n}}\left(2 \beta_{1,3}+2 \beta_{2,3} m^{2}\right) u_{12,0}^{2} u_{3,0}^{2} d x\right. \\
& \left.\quad+\mu_{3} \int_{\mathbb{R}^{n}} u_{3,0}^{4} d x\right),
\end{aligned}
$$

then, by (6.7)-(6.9), we have

$$
\begin{aligned}
& c_{2}(\mu) \leq E_{\mu}\left(t(\mu) u_{12,0}, t(\mu) k u_{12,0}, t(\mu) u_{3,0}\right) \\
= & \frac{1}{4} t^{2}(\mu)\left(\left(1+m^{2}\right) \int_{\mathbb{R}^{n}}\left(\left|\nabla u_{12,0}\right|^{2}+u_{12,0}^{2}\right) d x+\int_{\mathbb{R}^{n}}\left(\left|\nabla u_{3,0}\right|^{2}+u_{3,0}^{2}\right) d x\right) \\
= & c_{3}\left(\mu_{1}\right) \\
& \quad-\frac{c_{3}\left(\mu_{1}\right) \int_{\mathbb{R}^{n}}\left|u_{12,0}\right|^{4} d x\left(\mu-\mu_{1}\right)}{\left(\left(\mu_{1}+\mu_{2} m^{4}+2 \beta_{1,2} m^{2}\right) \int_{\mathbb{R}^{n}} u_{12,0}^{4} d x+\int_{\mathbb{R}^{n}}\left(2 \beta_{1,3}+2 \beta_{2,3} m^{2}\right) u_{12,0}^{2} u_{3,0}^{2} d x+\mu_{3} \int_{\mathbb{R}^{n}} u_{3,0}^{4} d x\right)} \\
& \quad+o\left(\left|\mu-\mu_{1}\right|^{2}\right) \\
= & c_{3}\left(\mu_{1}\right)-\frac{1}{4} \int_{\mathbb{R}^{n}}\left|u_{12,0}\right|^{4} d x\left(\mu-\mu_{1}\right)+o\left(\left|\mu-\mu_{1}\right|^{2}\right) .
\end{aligned}
$$

Thus

$$
\frac{c_{2}(\mu)-c_{2}\left(\mu_{1}\right)}{\mu-\mu_{1}} \geq-\frac{1}{4} \int_{\mathbb{R}^{n}}\left|u_{12,0}\right|^{4} d x+o\left(\left|\mu-\mu_{1}\right|^{2}\right) \quad \text { as } \mu \nearrow \mu_{1}
$$

so

$$
c_{2}^{\prime}\left(\mu_{1}\right) \geq-\frac{1}{4} \int_{\mathbb{R}^{n}}\left|u_{12,0}\right|^{4} d x
$$

Similarly,

$$
\begin{aligned}
& \frac{c_{2}(\mu)-c_{2}\left(\mu_{1}\right)}{\mu-\mu_{1}} \leq-\frac{1}{4} \int_{\mathbb{R}^{n}}\left|u_{12,0}\right|^{q} d x+o\left(\left|\mu-\mu_{1}\right|^{2}\right) \quad \text { as } \mu \searrow \mu_{1}, \\
& c_{2}^{\prime}\left(\mu_{1}\right) \leq-\frac{1}{4} \int_{\mathbb{R}^{n}}\left|u_{12,0}\right|^{4} d x .
\end{aligned}
$$

Hence

$$
c_{2}^{\prime}\left(\mu_{1}\right)=-\frac{1}{4} \int_{\mathbb{R}^{n}}\left|u_{12,0}\right|^{4} d x .
$$

Since $\left(d_{1} w, k d_{1} w, d_{3} w\right)$ is the ground state solution of (2.1), we have

$$
c_{2}^{\prime}\left(\mu_{1}\right)=-\frac{d_{1}^{4}}{4} \int_{\mathbb{R}^{n}} w^{4} d x
$$

Thus,

$$
\int_{\mathbb{R}^{n}}\left|u_{12,0}\right|^{4} d x=d_{1}^{4} \int_{\mathbb{R}^{n}} w^{4} d x
$$


By the similar arguments as above, that is, by computing $c_{3}^{\prime}\left(\mu_{2}\right), c_{3}^{\prime}\left(\mu_{3}\right)$, we obtain

$$
\int_{\mathbb{R}^{n}}\left|u_{3,0}\right|^{4} d x=d_{3}^{4} \int_{\mathbb{R}^{n}}|w|^{4} d x .
$$

Thus, we get (6.6b). Similarly, by computing $c_{3}^{\prime}\left(\beta_{i, j}\right)$, we obtain

$$
\int_{\mathbb{R}^{n}}\left|u_{12,0}\right|^{2}\left|u_{3,0}\right|^{2} d x=d_{1}^{2} d_{3}^{2} \int_{\mathbb{R}^{n}}|w|^{4} d x
$$

Thus, by (6.6a), (6.6c), we have

$$
\int_{\mathbb{R}^{n}}\left|u_{12,0}\right|^{2}\left|u_{3,0}\right|^{2} d x=d_{1}^{-2} d_{3}^{2} \int_{\mathbb{R}^{n}}\left|u_{12,0}\right|^{4} d x .
$$

Next, we prove

$$
\left(u_{12,0}, m u_{12,0}, u_{3,0}\right)=\left(d_{1} w, m d_{1} w, d_{3} w\right) .
$$

Since $\left(d_{1} w, m d_{1} w, d_{3} w\right)$ is a ground state solution of (2.1), we have

$$
\left\{\begin{array}{l}
\left(\mu_{1}+\beta_{1,2} m^{2}\right) d_{1}^{2}+\beta_{1,3} d_{3}^{2}=1 \\
\left(\mu_{2} m^{2}+\beta_{2,1}\right) d_{1}^{2}+\beta_{2,3} d_{3}^{2}=1 \\
\left(\beta_{3,1}+\beta_{3,2} m^{2}\right) d_{1}^{2}+\mu_{3} d_{3}^{2}=1
\end{array}\right.
$$

Let

$$
\left(u_{12}, m u_{12}, u_{3}\right)=\left(\frac{u_{12,0}}{d_{1}}, \frac{m u_{12,0}}{d_{1}}, \frac{u_{3,0}}{d_{3}}\right)
$$

then, by (6.10) and (6.11), we have

$$
\begin{aligned}
& \int_{\mathbb{R}^{n}}\left(\left|\nabla u_{12}\right|^{2}+u_{12}^{2}\right) d x \\
= & \frac{1}{d_{1}^{2}} \int_{\mathbb{R}^{n}}\left(\left|\nabla u_{12,0}\right|^{2}+u_{12,0}^{2}\right) d x \\
= & \frac{1}{d_{1}^{2}} \int_{\mathbb{R}^{n}}\left(\left(\mu_{1}+\beta_{1,2} m^{2}\right) u_{12,0}^{4}+\beta_{1,3} u_{12,0}^{2} u_{3,0}^{2}\right) d x \\
= & \frac{1}{d_{1}^{2}}\left(\mu_{1}+\beta_{1,2} m^{2}+\beta_{1,3} d_{1}^{-2} d_{3}^{2}\right) \int_{\mathbb{R}^{n}}\left|u_{12,0}\right|^{4} d x \\
= & \frac{1}{d_{1}^{4}} \int_{\mathbb{R}^{n}}\left|u_{12,0}\right|^{4} d x=\int_{\mathbb{R}^{n}}\left|u_{12}\right|^{4} d x .
\end{aligned}
$$

Similar, we have

$$
\int_{\mathbb{R}^{n}}\left(\left|\nabla u_{3}\right|^{2}+u_{3}^{2}\right) d x=\int_{\mathbb{R}^{n}}\left|u_{3}\right|^{4} d x
$$


Hence,

$$
\begin{aligned}
& \int_{\mathbb{R}^{n}}\left(\left|\nabla u_{12}\right|^{2}+u_{12}^{2}\right) d x \geq \int_{\mathbb{R}^{n}}\left(|\nabla w|^{2}+w^{2}\right) d x, \\
& \int_{\mathbb{R}^{n}}\left(\left|\nabla u_{3}\right|^{2}+u_{3}^{2}\right) d x \geq \int_{\mathbb{R}^{n}}\left(|\nabla w|^{2}+w^{2}\right) d x .
\end{aligned}
$$

Since $\left(u_{12,0}, m u_{12,0}, u_{3,0}\right)$ and $\left(d_{1} w, m d_{1} w, d_{3} w\right)$ are both the ground state solution of (2.1), we obtain

$$
\begin{aligned}
& \frac{1}{4}\left(\left(1+m^{2}\right) d_{1}^{2}+d_{3}^{2}\right) \int_{\mathbb{R}^{n}}\left(|\nabla w|^{2}+w^{2}\right) d x \\
= & \frac{1}{4} \int_{\mathbb{R}^{n}}\left(1+m^{2}\right)\left(\left|\nabla u_{12,0}\right|^{2}+u_{12,0}^{2}\right)+\left(\left|\nabla u_{3,0}\right|^{2}+u_{3,0}^{2}\right) d x \\
= & \frac{1}{4}\left[\left(1+m^{2}\right) d_{1}^{2} \int_{\mathbb{R}^{n}}\left(\left|\nabla u_{12}\right|^{2}+u_{12}^{2}\right) d x+d_{3}^{2} \int_{\mathbb{R}^{n}}\left(\left|\nabla u_{3}\right|^{2}+u_{3}^{2}\right) d x\right] \\
\geq & \frac{1}{4}\left(\left(1+m^{2}\right) d_{1}^{2}+d_{3}^{2}\right) \int_{\mathbb{R}^{n}}\left(|\nabla w|^{2}+w^{2}\right) d x,
\end{aligned}
$$

which implies that

$$
\begin{aligned}
& \int_{\mathbb{R}^{n}}\left(\left|\nabla u_{12}\right|^{2}+u_{12}^{2}\right) d x=\int_{\mathbb{R}^{n}}\left(|\nabla w|^{2}+w^{2}\right) d x, \\
& \int_{\mathbb{R}^{n}}\left(\left|\nabla u_{3}\right|^{2}+u_{3}^{2}\right) d x=\int_{\mathbb{R}^{n}}\left(|\nabla w|^{2}+w^{2}\right) d x .
\end{aligned}
$$

So, $u_{12}, u_{3}$ are positive ground state solutions of $(1.5)$. Since $\left(u_{12,0}, m u_{12,0}, u_{3,0}\right)$ satisfies (2.1) and $\left(\mu_{1}+\beta_{1,2} m^{2}\right) d_{1}^{2}+\beta_{1,3} d_{3}^{2}=1$, we have

$$
-\Delta u_{12}+u_{12}=\left(\mu_{1}+\beta_{1,2} m^{2}\right) d_{1}^{2} u_{12}^{3}+\beta_{3,1} d_{3}^{2} u_{3}^{2} u_{12}=u_{12}^{3} .
$$

So

$$
u_{12}^{3}=u_{3}^{2} u_{12} \text { and } \quad u_{3}=u_{12} \text {. }
$$

Since (1.5) has a unique positive ground state solution $w$, thus

$$
\left(u_{12,0}, m u_{12,0}, u_{3,0}\right)=\left(d_{1} w, m d_{1} w, d_{3} w\right) .
$$

This completes the proof.

\section{Acknowledgements}

This paper was completed when M. Zhen was visiting the University of British Columbia. He is grateful to the members in the department of Mathematics at University of British Columbia for their invitation and hospitality. The research of J. Wei is partially supported by NSERC of Canada. The first author was partially supported by NSERC of Canada. 


\section{References}

[1] B. Abdellaoui, V. Felli and I. Peral, Some remarks on systems of elliptic equations doubly critical the whole $\mathbb{R}^{N}$, Calc. Var. PDEs, 34 (2009), 97-137.

[2] A. Ambrosetti and E. Colorado, Bound and ground states of coupled nonlinear Schrödinger equations, C. R. Math. Acad. Sci. Paris, 342 (2006), 453-458.

[3] A. Ambrosetti and E. Colorado, Standing waves of some coupled nonlinear Schrödinger equations, J. Lond. Math. Soc., 75 (2007), 67-83.

[4] T. Bartsch, L. Jeanjean and N. Soave, Normalized solutions for a system of coupled cubic Schrödinger equations on $\mathbb{R}^{3}$, J. Math. Pures Appl., 106 (2016), 583-614.

[5] T. Bartsch and N. Soave, A natural constraint approach to normalized solutions of nonlinear Schrödinger equations and systems, J. Funct. Anal., 272 (2017), 4304-4333.

[6] T. Bartsch and N. Soave, Multiple normalized solutions for a competing system of Schrödinger equations, Calc. Var. Partial Differential Equations, 58 (2019).

[7] H. Berestycki, S. Terracini, K. Wang and J. C. Wei, On entire solutions of an elliptic system modeling phase seperations, Adv. Math., 243 (2013), 102-126.

[8] T. Bartsch, Z. Q. Wang and J. C. Wei, Bound states for a coupled Schrödinger system, J. Fixed Point Theory Appl., 2 (2007), 353-367.

[9] Z. Chen and W. Zou, An optimal constant for the existence of least energy solutions of a coupled Schrödinger system, Calc. Var. Partial Differential Equations, 48 (2013), 695-711.

[10] Z. Chen and W. Zou, Positive least energy solutions and phase separation for coupled Schrödinger equations with critical exponent, Arch. Ration. Mech. Anal., 205 (2012), 515551.

[11] Z. Chen and W. Zou, Positive least energy solutions and phase separation for coupled Schrödinger equations with critical exponent: higher dimensional case, Calc. Var. Partial Differential Equations, 52 (2015), 423-467.

[12] R. Frank and E. Lenzmann, Uniqueness of non-linear ground states for fractional Laplacians in $\mathbb{R}$, Acta Math., 210 (2013), 261-318.

[13] R. Frank, E. Lenzmann and L. Silvestre, Uniqueness of radial solutions for the fractional Laplacian, Commun. Pure Appl. Math., 69 (2016), 1671-1726.

[14] Y. Guo, S. Luo and W. Zou, The existence, uniqueness and nonexistence of the ground state to the $N$-coupled Schrödinger systems in $\mathbb{R}^{4},(n \leq 4)$, Nonlinearity, 31 (2018), 314-339.

[15] M. Kwong, Uniqueness of positive solutions of $\Delta u-u+u^{p}=0$ in $\mathbb{R}^{n}$, Arch. Ration. Mech. Anal., 105 (1989), 243-266.

[16] T. Lin and J. Wei, Ground state of $N$ coupled nonlinear Schrödinger equation in $\mathbb{R}^{n}, n \leq 3$, Commun. Math. Phys., 255 (2005), 629-653.

[17] Z. Liu and Z. Wang, Ground states and bound states of a nonlinear Schrödinger system, Adv. Nonlinear Study, 10 (2010), 175-193.

[18] T. Lin and J. Wei, Spikes in two coupled nonlinear Schrödinger equations, Ann. Inst. H. Poincaré Anal. Non Linéaire, 229 (2006), 538-569.

[19] T. Lin and J. Wei, Spikes in two-component systems of nonlinear Schrödinger equations with trapping potential J. Differential Equations, 22 (2005), 403-439.

[20] S. Peng and Z. Wang, Segregated and synchronized vector solutions for nonlinear Schrödinger systems, Arch. Rational. Mech. Anal., 208 (2013), 305-339.

[21] S. Peng, Q. Wang and Z. Wang, On coupled nonlinear Schrödinger systems with mixed couplings, Trans. Amer. Math. Soc., 371(11) (2019), 7559-7583.

[22] S. Peng, Y. Peng and Z. Wang, On critical systems with Sobolev critical growth, Calc. Var., 
55 (2016), 142.

[23] N.Soave and H. Tavares, New existence and symmetry results for least energy positive solutions of nonlinear Schrödinger system with mixed competition and cooperation terms, J. Differential Equations, 261 (2016), 505-537.

[24] B. Sirakov, Least energy solitary waves for a system of nonlinear Schrödinger equations in $\mathbb{R}^{n}$, Commum. Math. Phys., 271 (2007), 199-221.

[25] J. Wei and T. Weth, Nonradial symmetric bound states for a system of two coupled Schrödinger equations, Rend. Lincei Mat. Appl., 18 (2007), 279-293.

[26] J. Wei and T. Weth, Radial solutions and phase separation in a system of two Schrödinger equations, Arch. Ration. Mech. Anal., 190 (2008), 83-106.

[27] J. Wei and W. Yao, Uniqueness of positive solutions to some coupled nonlinear Schrödinger equations, Commun. Pure Appl. Anal., 11 (2012), 1003-1011.

[28] J. Wei and Y. Wu, Ground state of nonlinear Schrödinger system with mixed couplings, J. Math. Pures Appl., 141 (2020), 50-88.

[29] M. Zhen, J. He and H. Xu, Critical system involving fractional Laplacian, Commun. Pure Appl. Anal., 18 (2019), 237-253. 